\title{
EXTENSION THEORY FOR ELLIPTIC PARTIAL DIFFERENTIAL OPERATORS WITH PSEUDODIFFERENTIAL METHODS
}

\author{
GERD GRUBB
}

\begin{abstract}
This is a short survey on the connection between general extension theories and the study of realizations of elliptic operators $A$ on smooth domains in $\mathbb{R}^{n}, n \geq 2$. The theory of pseudodifferential boundary problems has turned out to be very useful here, not only as a formulational framework, but also for the solution of specific questions. We recall some elements of that theory, and show its application in several cases (including new results), namely to the lower boundedness question, and the question of spectral asymptotics for differences between resolvents.
\end{abstract}

\section{INTRODUCTION}

The general theory of extensions of a symmetric operator (or a dual pair of operators) in a Hilbert space, originating in the mid-1900's, has been applied in numerous works to ordinary differential equations (ODE), and also in a (smaller) number of works to partial differential equations (PDE).

There is a marked difference between the two cases: In ODE, the playground for boundary conditions is usually finite-dimensional vector spaces, where linear conditions can be expressed by the help of matrices. Moreover, the domains of differential operators defined by closure in $L_{2}$-based Hilbert spaces can usually all be expressed in terms of functions with the relevant number of absolutely continuous derivatives.

In contrast, boundary conditions for PDE (in space dimensions $n \geq 2$ ) are prescribed on infinite-dimensional vector spaces. Moreover, the domains of differential operators in $L_{2}$-based spaces will contain functions with distribution derivatives, not continuous and possibly highly irregular.

Whereas extensions of ODEs can usually be described in terms of matrices, the tools to interpret extensions in PDE cases are therefore much more complicated. We shall give a survey of some tools developed through the years, and their applications, emphasizing the use of pseudodifferential operators.

Outline. In Section 2 we recall the basic issues of elliptic boundary value problems. Pseudodifferential operators ( $\psi$ do's) are introduced in Section 3, and Section 4 introduces pseudodifferential boundary operators ( $\psi$ dbo's). In Section 5 we recall the elements of a general abstract extension theory, and in Section 6 we show how this is implemented for realizations $\widetilde{A}$ of an elliptic operator $A$ on a domain $\Omega \subset \mathbb{R}^{n}$. Section 7 focuses on the resolvent formulas that can be obtained via the general theory. In the last sections we go through several cases where pseudodifferential techniques have proved extremely useful (some of the results here are quite recent): In Section 8 it is the question of whether lower boundedness holds simultaneously

1991 Mathematics Subject Classification. 35J40, 47G30, 58C40.

Key words and phrases. Elliptic operators; pseudodifferential boundary operators; extension theory; lower bounds; unbounded domains; exterior problems; singular Green operators; spectral asymptotics . 
for a realization $\widetilde{A}$ and the operator $L$ over the boundary that enters in the corresponding boundary condition; the new results deal with unbounded domains. In Section 9 it is the question of showing Weyl-type spectral asymptotics formulas for differences between resolvents. The results there go back to the early theory, and Section 10 presents some additional new results.

\section{Elliptic Boundary Value problems}

In the following we use the customary multi-index notation for differential operators: $\partial=\partial_{x}=\left(\partial_{1}, \ldots, \partial_{n}\right), \partial_{j}=\partial_{x_{j}}=\partial / \partial x_{j}$, and $D=D_{x}=\left(D_{1}, \ldots, D_{n}\right)$, $D_{j}=D_{x_{j}}=-i \partial / \partial x_{j}$; then $\partial^{\alpha}=\partial_{1}^{\alpha_{1}} \cdots \partial_{n}^{\alpha_{n}}, D^{\alpha}=D_{1}^{\alpha_{1}} \cdots D_{n}^{\alpha_{n}}$, for $\alpha \in \mathbb{N}_{0}^{n}$; here $|\alpha|=\alpha_{1}+\cdots+\alpha_{n}$.

A differential operator of order $m>0$,

$$
A=\sum_{|\alpha| \leq m} a_{\alpha}(x) D^{\alpha}
$$

is said to be elliptic, resp. strongly elliptic, on a set $U \subset \mathbb{R}^{n}$, when the principal symbol

$$
a_{m}(x, \xi)=\sum_{|\alpha|=m} a_{\alpha}(x) \xi^{\alpha}
$$

satisfies

$$
a_{m}(x, \xi) \neq 0, \text { resp. } \operatorname{Re} a_{m}(x, \xi)>0,
$$

for $x \in U, \xi \in \mathbb{R}^{n} \backslash\{0\}$. As a basic example, the Laplacian $\Delta=\partial_{1}^{2}+\cdots+\partial_{n}^{2}$ has principal symbol (and symbol) equal to $-|\xi|^{2}$, so it is elliptic, and $-\Delta$ is strongly elliptic. The Laplacian has been studied for several hundred years, and the problems around it solved by explicit solution formulas. It is the cases with variable $(x-$ dependent) coefficients, and domains more general than simple geometric figures, that have been a challenge in more modern times.

The problem

$$
A u=f
$$

for a given function $f$ on a subset $\Omega$ of $\mathbb{R}^{n}$ usually has infinitely many solutions. To get a problem with unique solvability, we must adjoin extra conditions such as suitable boundary conditions. We can consider $A$ with a domain consisting of the functions satisfying the boundary condition, as an operator $\widetilde{A}$ acting between suitable spaces.

Then the question of existence of a solution corresponds to the question of whether $\widetilde{A}$ is surjective, and the question of uniqueness of a solution corresponds to the question of whether $\widetilde{A}$ is injective. In this way, the question of solvability of differential equations is turned into a question of properties of specific operators. The operator point of view became particularly fruitful when it was combined with appropriate scales of function spaces, such as the Sobolev spaces, Sobolev [So50], and with Distribution theory, Schwartz [Sch50].

When $\Omega$ is a smooth open subset of $\mathbb{R}^{n}$ with boundary $\partial \Omega=\Sigma$, we refer to the standard $L_{2}$-Sobolev spaces, with the following notation: $H^{s}\left(\mathbb{R}^{n}\right)(s \in \mathbb{R})$ has the norm $\|v\|_{s}=\left\|\mathcal{F}^{-1}\left(\langle\xi\rangle^{s} \mathcal{F} v\right)\right\|_{L_{2}\left(\mathbb{R}^{n}\right)}$; here $\mathcal{F}$ is the Fourier transform

$$
\mathcal{F}: u(x) \mapsto(\mathcal{F} u)(\xi)=\hat{u}(\xi)=\int_{\mathbb{R}^{n}} e^{-i x \cdot \xi} u(x) d x,
$$

and $\langle\xi\rangle=\left(1+|\xi|^{2}\right)^{\frac{1}{2}}$. Next, with $r_{\Omega}$ denoting restriction to $\Omega$,

$$
H^{s}(\Omega)=r_{\Omega} H^{s}\left(\mathbb{R}^{n}\right),
$$


provided with the norm $\|u\|_{s}=\inf \left\{\|v\|_{s} \mid v \in H^{s}\left(\mathbb{R}^{n}\right), u=r_{\Omega} v\right\}$. Moreover,

$$
H_{0}^{s}(\bar{\Omega})=\left\{u \in H^{s}\left(\mathbb{R}^{n}\right) \mid \operatorname{supp} u \subset \bar{\Omega}\right\},
$$

closed subspace of $H^{s}\left(\mathbb{R}^{n}\right)$; it identifies with the antidual space of $H^{-s}(\Omega)$ (the space of antilinear, i.e., conjugate linear, functionals), with a duality consistent with the $L_{2}$ scalar product. For $s$ equal to a nonnegative integer $k, H_{0}^{k}(\bar{\Omega})$ is usually written $H_{0}^{k}(\Omega)$. Spaces over the boundary, $H^{s}(\Sigma)$, are defined by local coordinates from $H^{s}\left(\mathbb{R}^{n-1}\right), s \in \mathbb{R}$. (There are many equally justified equivalent choices of norms there; one can choose a particular norm when convenient.) When $s>0$, there are dense continuous embeddings

$$
H^{s}(\Sigma) \subset L_{2}(\Sigma) \subset H^{-s}(\Sigma),
$$

and there is an identification of $H^{-s}(\Sigma)$ with the antidual space of $H^{s}(\Sigma)$, such that the duality $(\varphi, \psi)_{-s, s}$ coincides with the $L_{2}(\Sigma)$-scalar product when the elements lie there. Detailed explanations are found in many books, e.g. Lions and Magenes [LM68], Hörmander [H63], [G09]. (There is a difference of notation: For $k \in \mathbb{N}$, the spaces denoted $H_{0}^{k+\frac{1}{2}}(\Omega)$ in [LM68] are not the same as our $H_{0}^{k+\frac{1}{2}}(\bar{\Omega})$ that are consistent with [H63]; they have the best duality and interpolation properties.)

Consider the case where $A$ is defined on a smooth open subset $\Omega$ of $\mathbb{R}^{n}$ and has coefficients in $C^{\infty}(\bar{\Omega})$, and assume that $A$ is elliptic on $\bar{\Omega}$. The results in this case are a model for results under weaker smoothness hypotheses. One defines the maximal realization $A_{\max }$ as the operator acting like $A$ in the distribution sense with domain

$$
D\left(A_{\max }\right)=\left\{u \in L_{2}(\Omega) \mid A u \in L_{2}(\Omega)\right\} ;
$$

it is a closed, unbounded operator in $L_{2}(\Omega)$. The minimal realization $A_{\min }$ is defined as the closure of $A$ acting on $C_{0}^{\infty}(\Omega)$ (the compactly supported $C^{\infty}$-functions on $\Omega$ ). When $\Omega$ is bounded, or is unbounded and there are suitable bounds on the coefficients of $A$,

$$
D\left(A_{\min }\right)=H_{0}^{m}(\Omega)
$$

The formal adjoint $A^{\prime}$ of $A$ is the differential operator acting as follows:

$$
A^{\prime} u=\sum_{|\alpha| \leq m} D^{\alpha}\left(\bar{a}_{\alpha}(x) u\right)
$$

By definition, $A_{\min }^{\prime}$ and $A_{\max }$ are adjoints of one another (as unbounded operators in $L_{2}(\Omega)$ ).

The linear operators $\widetilde{A}$ satisfying

$$
A_{\min } \subset \widetilde{A} \subset A_{\max }
$$

are called realizations of $A$.

Generally, $A_{\max }$ is far from being injective, whereas $A_{\min }$ is far from being surjective, but it may be possible to find realizations $\widetilde{A}$ that are bijective from $D(\widetilde{A})$ to $L_{2}(\Omega)$.

We see that the theory of distributions (which in this context was preceded by the definition of differential operators acting in the weak sense) allows defining operators representing the action of $A$ in a generalized sense. Here invertibility can sometimes be achieved by methods of functional analysis. A fundamental example is the Dirichlet problem (where $\gamma_{0} u=\left.u\right|_{\Sigma}$ )

$$
A u=f \text { in } \Omega, \quad \gamma_{0} u=\varphi \text { on } \Sigma,
$$

for a strongly elliptic second-order operator having $\operatorname{Re}(A v, v) \geq c\|v\|_{L_{2}(\Omega)}^{2}$ with $c>0$ for $v \in C_{0}^{\infty}(\Omega)$. By use of the so-called Lax-Milgram lemma one could define a realization $A_{\gamma}$ of $A$ with $D\left(A_{\gamma}\right) \subset H_{0}^{1}(\Omega)$, such that $A_{\gamma}: D\left(A_{\gamma}\right) \rightarrow L_{2}(\Omega)$ bijectively. (Details are found in many books, e.g. [G09], Ch. 12.) 
But then the question was: How close is $A_{\gamma}^{-1}$ to solving the problem in a more classical sense? Second-order derivatives have a meaning on $H^{2}(\Omega)$, by closure of the definition on $C^{2}(\bar{\Omega})$, so one can ask:

- If $f \in L_{2}(\Omega)$, is $u \in H^{2}(\Omega)$ ?

- More generally, if $f \in H^{k}(\Omega)$ for some $k \in \mathbb{N}_{0}$, is $u \in H^{k+2}(\Omega)$ ?

The answer was first found for the behavior of $u$ in the interior of $\Omega$ : Indeed, when $f \in H^{k}(\Omega), u$ is in $H^{k+2}$ over subsets of $\Omega$ with positive distance from the boundary. This is the so-called interior regularity.

There remained the question of regularity at the boundary. It was answered positively in papers by Nirenberg [N55] and Ladyzhenskaya (see the account in [L85]). This was followed up by research on higher-order operators $A$ and more general boundary conditions $T u=\varphi$ (possibly vector valued), where results on interior regularity and regularity at the boundary were established under suitable conditions. Besides ellipticity of the operator $A$ one needs a condition on how the boundary condition fits together with $A$. Some authors called it the "covering condition" or the "complementing condition", but the name "the Shapiro-Lopatinskiu condition" (after [Sh53], [L53]) has been more generally used. It is also customary to call the system $\{A, T\}$ elliptic when it holds (this was suggested in Hörmander [H63]; we return to a motivation in Section 4). A fundamental reference in this connection is the paper of Agmon, Douglis and Nirenberg [ADN59] that collects and expands the knowledge on elliptic boundary value problems. An important point of view was to obtain so-called "à priori estimates" (estimates of a Sobolev norm on $u$ by norms on $A u$ and $T u$ plus a lower order norm on $u$ ), shown for smooth functions at first, and extended to the considered solution.

Important monographs exposing the theories and the various authors' own contributions were written by Agmon [A65], Lions and Magenes [LM68]; the latter moreover contains valuable information on the surrounding literature. The early theory is exposed in Courant and Hilbert I-II [CH53], [CH62].

In the works at that time, although the striving to show existence of a solution operator was always in the picture, the emphasis was more on showing qualitative properties of the unknown function $u$ in terms of properties of the given data $f$ and $\varphi$, regardless of whether $u$ could be described by an operator acting on $\{f, \varphi\}$ or not.

Direct machinery to construct approximate solution operators in general came into the picture with the advent of pseudodifferential methods.

\section{Pseudodifferential operators}

One of the few cases where an elliptic differential operator has an explicit solution operator is the case of $I-\Delta$ on $\mathbb{R}^{n}$, whose action can be described by use of the Fourier transform $\mathcal{F}$ as $(1-\Delta) u=\mathcal{F}^{-1}\left(\left(1+|\xi|^{2}\right) \mathcal{F} u\right)$, and whose solution operator is

$$
\text { Op }\left(\frac{1}{1+|\xi|^{2}}\right) u=\mathcal{F}^{-1}\left(\frac{1}{1+|\xi|^{2}} \mathcal{F} u\right)
$$

A variable-coefficient elliptic differential operator on $\mathbb{R}^{n}$ can also be described by the help of the Fourier transform,

$$
A u=\sum_{|\alpha| \leq m} a_{\alpha}(x) D^{\alpha} u=\sum_{|\alpha| \leq m} a_{\alpha}(x) \mathcal{F}^{-1}\left(\xi^{\alpha} \mathcal{F} u\right)=\mathcal{F}^{-1} a(x, \xi) \mathcal{F} u=\operatorname{Op}(a(x, \xi)) u
$$

where $a(x, \xi)=\sum_{|\alpha| \leq m} a_{\alpha}(x) \xi^{\alpha}$ is the symbol. But even when the symbol satisfies $a(x, \xi) \neq 0$ for all $x, \xi$, the operator

$$
\mathrm{Op}\left(a(x, \xi)^{-1}\right)=\mathcal{F}^{-1} a(x, \xi)^{-1} \mathcal{F}
$$


is not an exact inverse. Nevertheless, it is useful in the discussion of solutions, since one can show that

$$
\mathrm{Op}(a(x, \xi)) \operatorname{Op}\left(a(x, \xi)^{-1}\right)=I+\mathcal{R},
$$

where the remainder $\mathcal{R}$ is of order -1 (lifts the exponent of a Sobolev space by 1 ).

A thorough treatment of $\operatorname{Op}\left(a(x, \xi)^{-1}\right)$ and suitable generalizations that come closer to being an inverse of $A$ (such "almost-inverses" are called parametrices) was obtained with the invention of pseudodifferential operators, $\psi$ do's. Some of the initiators were Mihlin [M48], Calderón and Zygmund [CZ57], Seeley [Se65], Kohn and Nirenberg [KN65], Hörmander [H65], [H71].

General $\psi$ do's are defined from general symbols $p(x, \xi)$ as

$$
\mathrm{Op}(p(x, \xi)) u=\mathcal{F}^{-1} p(x, \xi) \mathcal{F} u=\int_{\mathbb{R}^{n}} e^{i x \cdot \xi} p(x, \xi) \hat{u}(\xi) d \xi,
$$

where we use the notation $d \xi=(2 \pi)^{-n} d \xi$; here $p(x, \xi)$ is required to belong to a suitable class of functions.

Not only do the operators make sense on $\mathbb{R}^{n}$ where the Fourier transform acts, they are also given a meaning on manifolds, by use of coordinate change formulas and cutoff functions. The theory is not altogether easy; it uses concepts from distribution theory in a refined way. Moreover, it is not exact but qualitative in many statements, so it can be something of a challenge to derive good results from its use. A fine achievement is that it leads to Fredholm operators, when applied to elliptic operators on compact manifolds. Here one is just a small step away from having invertible operators; this can sometimes be achieved by relying on additional knowledge of the situation.

A so-called "classical" $\psi$ do is an operator defined from a symbol that has an asymptotic series expansion in homogeneous terms (a polyhomogeneous symbol):

$$
p(x, \xi) \sim \sum_{j \in \mathbb{N}_{0}} p_{m-j}(x, \xi), \quad p_{m-j}(x, t \xi)=t^{m-j} p_{m-j}(x, \xi) \text { for }|\xi| \geq 1, t \geq 1 .
$$

It is said to be of order $m$, and $\operatorname{Op}(p)$ maps $H^{s}$ to $H^{s-m}$ for all $s \in \mathbb{R}$. $p_{m}$ is called the principal symbol, and $p$ is said to be elliptic when $p_{m}(x, \xi) \neq 0$ for $|\xi| \geq 1$. Here one has that

$$
\mathrm{Op}(p) \mathrm{Op}\left(p^{\prime}\right)=\mathrm{Op}\left(p p^{\prime}\right)+\mathcal{R}_{1}=\mathrm{Op}\left(p_{m} p_{m^{\prime}}^{\prime}\right)+\mathcal{R}_{2},
$$

where $\mathcal{R}_{1}$ and $\mathcal{R}_{2}$ are of order $m+m^{\prime}-1$. In this way, the principal part dominates the behavior. When $p$ is elliptic, the principal part of a parametrix is found as $p_{m}^{-1}$ (for $|\xi| \geq 1$, extended smoothly to $\xi \in \mathbb{R}^{n}$ ). Also the notation $p^{0}$ is used for the principal symbol.

There exist many different symbol classes with generalizations of the above properties, designed for particular purposes.

The very attractive feature of classical $\psi$ do's is that they form a scale of operators of all integer orders, including differential operators among those of positive order, and including parametrices and inverses of elliptic differential operators among those of negative order. Moreover, it is an "algebra", in the sense that the elements by composition (and by addition) lead to other classical $\psi$ do's.

The calculus is explained in the original papers and in several subsequent books, such as Treves [T80], Hörmander [H85]; a detailed introduction can be also found in Chapters 7-8 of [G09].

The $\psi$ do theory gives (after one has done the work to set it up) an easy proof of interior regularity of solutions to elliptic problems. 


\section{Pseudodifferential Boundary operators}

When an elliptic differential operator $A$ is considered on a subset of $\mathbb{R}^{n}$ or on a manifold with boundary — let us here for simplicity just consider the case of a smooth bounded open subset $\Omega$ of $\mathbb{R}^{n}$ — we must impose boundary conditions to get uniquely solvable problems. Let assume that we are in a case where the boundary condition $T u=\varphi$ together with (1) gives a uniquely solvable problem; here $T$ is a trace operator mapping functions on $\Omega$ into $M$-tuples of functions on $\Sigma=\partial \Omega$. We can formulate this in terms of matrices:

$$
\left(\begin{array}{c}
A \\
T
\end{array}\right): C^{\infty}(\bar{\Omega}) \rightarrow \underset{C^{\infty}(\Sigma)^{M}}{\times} \text { has an inverse }\left(\begin{array}{ll}
R & K
\end{array}\right): \underset{C^{\infty}(\Sigma)^{M}}{\times} \underset{C^{\infty}(\bar{\Omega})}{\times} \rightarrow C^{\infty}(\bar{\Omega}) .
$$

Here $K$ is called a Poisson operator; it solves the semi-homogeneous problem

$$
A v=0 \text { in } \Omega, \quad T v=\varphi \text { on } \Sigma .
$$

The operator $R$ solves the other semi-homogeneous problem

$$
A w=f \text { in } \Omega, \quad T w=0 \text { on } \Sigma .
$$

In a closer analysis of $R$, we can write it as a sum of two terms:

$$
R=Q_{+}+G \text {, }
$$

where $Q$ is the $\psi$ do $A^{-1}$ on $\mathbb{R}^{n}, Q_{+}$is its truncation $r^{+} Q e^{+}$to $\Omega$, and $G$ is a supplementing operator adapted to the specific boundary condition, called a singular Green operator (s.g.o.). The operator $e^{+}$stands for extension by 0 (to functions on $\left.\mathbb{R}^{n}\right)$, and the operator $r^{+}$stands for restriction to $\Omega$.

The calculus of pseudodifferential boundary operators ( $\psi$ dbo's) was initated by Boutet de Monvel [B71], who introduced operator systems encompassing both the systems $\left(\begin{array}{l}A \\ T\end{array}\right)$ and their solution operators $\left(\begin{array}{ll}R & K\end{array}\right)$. The original presentation is somewhat brief, and was followed up by extended expositions, in the detailed book of Rempel and Schulze [RS82], which elaborated the index theory, and in the paper [G84] which completed some proofs of composition rules (with new points of view), and showed spectral asymptotic estimates for singuar Green operators. The book [G96], whose first edition was issued in 1986, developed a calculus of parameterdependent $\psi$ dbo's, leading to resolvent and heat operator constructions. The recent book [G09] gives a full introduction to the theory.

In the systematic calculus of Boutet de Monvel one consider systems (called Green operators):

$$
\mathcal{A}=\left(\begin{array}{cc}
P_{+}+G & K \\
T & S
\end{array}\right): \begin{gathered}
C^{\infty}(\bar{\Omega})^{N} \\
C^{\infty}(\Sigma)^{M}
\end{gathered} \rightarrow \begin{gathered}
C^{\infty}(\bar{\Omega})^{N^{\prime}} \\
C^{\infty}(\Sigma)^{M^{\prime}}
\end{gathered}, \text { where }
$$

- $P$ is a ps.d.o. on $\mathbb{R}^{n}$, satisfying the so-called transmission condition at $\Sigma$ (always true for operators stemming from elliptic differential operators);

- $P_{+}=r^{+} P e^{+}$is the truncation to $\Omega$ (the transmission condition assures that $P_{+}$maps $C^{\infty}(\bar{\Omega})$ into $\left.C^{\infty}(\bar{\Omega})\right)$;

- $T$ is a trace operator from $\Omega$ to $\Sigma, K$ is a Poisson operator from $\Sigma$ to $\Omega, S$ is a $\psi$ do on $\Sigma$;

- $G$ is a singular Green operator, e.g. of the type $K T$.

The composition of two such systems is again a system belonging to the calculus.

The operators extend to act on Sobolev spaces. For $T$ and $G$ there is a condition expressing which differential trace operators $\gamma_{j} u=\left.(\partial / \partial n)^{j} u\right|_{\Sigma}$ that enter: $T$ or $G$ is said to be of class $r$ when only $\gamma_{j}$ 's with $j<r$ enter; and then they act on $H^{s}(\Omega)$ 
for $s>r-\frac{1}{2}$. The class 0 case is the case where they are purely integral operators, well-defined on $L_{2}(\Omega)$.

All entries can be matrix-formed. They are defined in local coordinates by formulas involving Fourier transformation and polyhomogeneous symbols. The idea is as follows: In local coordinates at the boundary, where $\Omega$ and $\Sigma$ are replaced by $\mathbb{R}_{+}^{n}=\left\{x \in \mathbb{R}^{n} \mid x_{n}>0\right\}$ and $\mathbb{R}^{n-1}$ (with points $x^{\prime}=\left(x_{1}, \ldots, x_{n-1}\right)$ ), the system has for each $\left(x^{\prime}, \xi^{\prime}\right)$ a boundary symbol operator acting in the $x_{n}$-variable:

$$
\begin{aligned}
& a\left(x^{\prime}, \xi^{\prime}, D_{n}\right)=
\end{aligned}
$$

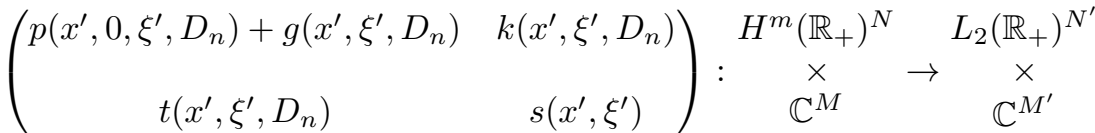

Here $m$ is the order of the operator. Each entry in a acts in a specific way. E.g., when the matrix is $\left(\begin{array}{l}A \\ T\end{array}\right)$ in (3), the boundary symbol operator is the model operator obtained by freezing the coefficients at $x^{\prime}$ and replacing derivatives in $D_{x^{\prime}}^{\alpha}$ by their Fourier transforms $\left(\xi^{\prime}\right)^{\alpha}$ (with respect to $x^{\prime} \in \mathbb{R}^{n-1}$ ). The principal boundary symbol operator $a^{0}\left(x^{\prime}, \xi^{\prime}, D_{n}\right)$ is formed of the top order terms. The principal boundary symbol operator for $(R \quad K)$ is the inverse of the principal boundary symbol operator for $\left(\begin{array}{c}A \\ T\end{array}\right)$. (For $(5), g$ and $t$ must be of class $\leq m$.)

From the boundary symbol operator one defines a full operator by applying the $\psi$ do definition in the $x^{\prime}$-variable,

$$
\mathrm{Op}^{\prime}\left(a\left(x^{\prime}, \xi^{\prime}, D_{n}\right)\right) u=\int e^{i x^{\prime} \cdot \xi^{\prime}} a\left(x^{\prime}, \xi^{\prime}, D_{n}\right)\left(\mathcal{F}_{y^{\prime} \rightarrow \xi^{\prime}} u\left(y^{\prime}, x_{n}\right)\right) d \xi^{\prime}
$$

The symbols have asymptotic series of terms that are homogeneous in $\left(\xi^{\prime}, \xi_{n}\right)$ (different rules apply to the different ingredients, and we must refer to the mentioned references for further details). One then defines $\mathcal{A}$ to be elliptic, when

(1) $P$ is elliptic, i.e. its principal symbol $p^{0}(x, \xi)$ is invertible at each $(x, \xi)$ with $|\xi| \geq 1$

(2) the principal boundary symbol operator $a^{0}\left(x^{\prime}, \xi^{\prime}, D_{n}\right)$ is invertible at each $\left(x^{\prime}, \xi^{\prime}\right)$ with $\left|\xi^{\prime}\right| \geq 1$.

For a system $\left(\begin{array}{l}A \\ T\end{array}\right)$ formed of an elliptic differential operator $A$ and a differential trace operator $T, 2)$ is precisely the old covering/complementing/Shapiro-Lopatinskiu condition for $\{A, T\}$.

In the elliptic case, one can construct a parametrix $\mathcal{B}^{0}$ from the inverses of the symbols in 1)-2), such that $\mathcal{A B}^{0}-I$ and $\mathcal{B}^{0} \mathcal{A}-I$ have order $\leq-1$, and the construction can be refined to give errors of arbitrarily low order. With supplementing information it can be possible to obtain an inverse.

For example, there holds a a solvability theorem for an elliptic differential operator problem as in (3), formulated in this framework as follows:

Theorem 4.1. Let $\Omega \subset \mathbb{R}^{n}$ be a smooth, bounded open set, denote $\partial \Omega=\Sigma$, and let $A=\sum_{|\alpha| \leq 2 m} a_{\alpha}(x) D^{\alpha}$ with $a_{\alpha} \in C^{\infty}(\bar{\Omega})$ be elliptic on $\bar{\Omega}$, i.e., $\sum_{|\alpha|=2 m} a_{\alpha}(x) \xi^{\alpha} \neq$ 0 for $x \in \bar{\Omega}, \xi \in \mathbb{R}^{n} \backslash\{0\}$. Let $T=\left(T_{j}\right)_{j=1}^{m}$ be a column vector of trace operators $T_{j}=\gamma_{0} B_{j}$, where the $B_{j}$ are differential operators of order $m_{j}$ with $C^{\infty}$-coefficients, $0 \leq m_{1}<\cdots<m_{m} \leq 2 m-1$. (Then $T$ is of class $r=m_{m}+1 \leq 2 m$.) Assume that $\{A, T\}$ is elliptic.

The operator $\mathcal{A}=\left(\begin{array}{l}A \\ T\end{array}\right)$ defines a continuous mapping

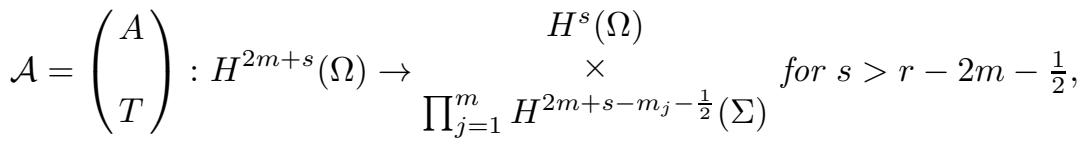


and there is a system $\mathcal{B}=\left(\begin{array}{ll}R & G\end{array}\right)$ (a parametrix) belonging to the calculus and continuous in the opposite direction, such that

$$
\begin{gathered}
\mathcal{A B}=\left(\begin{array}{ll}
I & 0 \\
0 & I
\end{array}\right)+\mathcal{R}_{1}, \quad \mathcal{B} \mathcal{A}=I+\mathcal{R}_{2}, \\
H^{s}(\Omega) \\
\mathcal{R}_{1}: \underset{\prod_{j=1}^{m} H^{2 m+s-m_{j}-\frac{1}{2}}(\Sigma)}{\times} \rightarrow \underset{\prod_{j=1}^{s^{\prime}}(\Omega)}{\times} H^{2 m+s^{\prime}-m_{j}-\frac{1}{2}}(\Sigma) \\
\mathcal{R}_{2}: H^{2 m+s}(\Omega) \rightarrow H^{2 m+s^{\prime}}(\Omega)
\end{gathered}
$$

for all $s$ as in (6), all $s^{\prime} \geq s$. Here $K$ is a row vector of Poisson operators $\left(K_{j}\right)_{j=1}^{m}$ of orders $-m_{j}$, and $R=Q_{+}+G$, where $Q$ is a parametrix of $A$ on a neighborhood of $\bar{\Omega}$, and $G$ is a singular Green operator.

The operator $\mathcal{A}$ in (6) is Fredholm for each s, with the same finite dimensional kernel and cokernel in $C^{\infty}$ for all $s$. $\mathcal{B})$.

If $\mathcal{A}$ is bijective, the inverse belongs to the calculus (and is of the same form as

When $r=2 m$, the lower limit for $s$ is $-\frac{1}{2}$; cases where it is $<-\frac{1}{2}$ occur for example for the Dirichlet problem, where $r=m$, and $s$ can go down to $-m-\frac{1}{2}$. It is useful to know that the Poisson operator $K$ in fact has the mapping property

$$
K: \prod_{j=1}^{m} H^{2 m+s-m_{j}-\frac{1}{2}}(\Sigma) \rightarrow H^{2 m+s}(\Omega)
$$

for all $s \in \mathbb{R}$. The trace operator $T$ is called normal, when $\gamma_{0} B_{j}=b_{j} \gamma_{m_{j}}+$ $\sum_{k<m_{j}} B_{j k} \gamma_{k}$ with an invertible coefficient $b_{j}$ for each $j$. (More general normal boundary value problems are described below in Section 9.)

For example, for a second-order strongly elliptic operator with a Dirichlet condition, the operator in the theorem maps as follows:

$$
\mathcal{A}=\left(\begin{array}{c}
A \\
\gamma_{0}
\end{array}\right): H^{2+s}(\Omega) \rightarrow \underset{H^{\frac{3}{2}+s}(\Sigma)}{\times} \underset{H^{s}(\Omega)}{\times} \text { for } s>-\frac{3}{2},
$$

with parametrices and solution operators continuous in the opposite direction.

Elliptic operators $A$ of odd order occur mainly as square matrix-formed operators, and there is a similar theorem for such cases, where also the $B_{j}$ can be matrix-formed. Operators of Dirac-type are a prominent first-order example. The matrix case is also interesting for even-order operators. The results can moreover be worked out for operators defined on manifolds, acting in vector bundles. (See e.g. [G74], on the even-order case, for notation and the appropriate definition of normal boundary conditions.)

\section{EXTENSION THEORIES}

We shall now recall some elements of the functional analysis theory of extensions of given operators. This has a long history, with prominent contributions from J. von Neumann in 1929 [N29], K. Friedrichs in 1934 [F34], M. G. Kreйn in 1947 [K47], M. I. Vishik in 1952 [V52], M. S. Birman in 1956 [B56], and others. The present author made a number of contributions in 1968-74 [G68]-[G74], completing the preceding theories and working out applications to elliptic boundary value problems; further developments are found e.g. in [G83], [G84], and in recent works.

At the same time there was another, separate development of abstract extension theories, where the operator concept gradually began to be replaced by the 
concept of relations. This development has been aimed primarily towards applications to ODE, however including operator-valued such equations and Schrödinger operators on $\mathbb{R}^{n}$; keywords in this connection are: boundary triples theory, WeylTitchmarsh $m$-functions and Kreĭn resolvent formulas. Cf. e.g. Kočuber [K75], Vainerman [V80], Lyantze and Storozh [LS83], Gorbachuk and Gorbachuk [GG91], Derkach and Malamud [DM91], Arlinskii [A99], Malamud and Mogilevskii [MM02], Brüning, Geyler and Pankrashkin [BGP06], and their references. In later years there have also been applications to elliptic boundary value problems, cf. e.g. Amrein and Pearson [AP04], N.D. Kopachevskiı̌ and S.G. Kreı̌n [KK04], Behrndt and Langer [BL07], Ryzhov [R07], Brown, Marletta, Naboko and Wood [BMNW08], Gesztesy and Mitrea [GM08], and their references.

The connection between the two lines of extension theories has been clarified in a recent work of Brown, Grubb and Wood [BGW09].

At this point we should also mention the recent efforts for problems on nonsmooth domains: Posilicano and Raimondi [PR09], Grubb [G08], Gesztesy and Mitrea [GM08, GM11], Abels, Grubb and Wood [AGW11]; here [G08, GM11, AGW11] use [G68].

In the following, we shall use the notation from [G68]-[G74] and [BGW09].

Let there be given a pair $A_{\min }, A_{\min }^{\prime}$ of closed, densely defined operators in a Hilbert space $H$, such that the following holds:

$$
A_{\min } \subset\left(A_{\min }^{\prime}\right)^{*}=: A_{\max }, \quad A_{\min }^{\prime} \subset\left(A_{\min }\right)^{*}=: A_{\max }^{\prime} .
$$

Let $\mathcal{M}=\left\{\widetilde{A} \mid A_{\min } \subset \widetilde{A} \subset A_{\max }\right\}$. Write $\widetilde{A} u$ as $A u$, when $\widetilde{A} \in \mathcal{M}$.

We assume that there is given an operator $A_{\gamma} \in \mathcal{M}$, the reference operator, with $0 \in \varrho\left(A_{\gamma}\right)$ (the resolvent set); then

$$
A_{\text {min }} \subset A_{\gamma} \subset A_{\max }, \quad A_{\text {min }}^{\prime} \subset A_{\gamma}^{*} \subset A_{\text {max }}^{\prime} .
$$

The case where $A_{\min }=A_{\min }^{\prime}$ and $A_{\gamma}$ is selfadjoint, is called the symmetric case.

Let $Z=\operatorname{ker} A_{\max }, Z^{\prime}=\operatorname{ker} A_{\max }^{\prime}$, and define the basic non-orthogonal decompositions

$$
D\left(A_{\max }\right)=D\left(A_{\gamma}\right) \dot{+} Z, \quad D\left(A_{\max }^{\prime}\right)=D\left(A_{\gamma}^{*}\right) \dot{+} Z^{\prime},
$$

denoted $u=u_{\gamma}+u_{\zeta}=\operatorname{pr}_{\gamma} u+\operatorname{pr}_{\zeta} u$, where $\operatorname{pr}_{\gamma}=A_{\gamma}^{-1} A_{\max }$, with a similar notation with primes.

By $\operatorname{pr}_{X} u=u_{X}$ we denote the orthogonal projection from $H$ to $X$. The injection $X \hookrightarrow H$ is denoted $\mathrm{i}_{X}$ (it is the adjoint of $\operatorname{pr}_{X}: H \rightarrow X$ ).

There holds an "abstract Green's formula" for $u \in D\left(A_{\max }\right), v \in D\left(A_{\max }^{\prime}\right)$ :

$$
(A u, v)-\left(u, A^{\prime} v\right)=\left((A u)_{Z^{\prime}}, v_{\zeta^{\prime}}\right)-\left(u_{\zeta},\left(A^{\prime} v\right)_{Z}\right) .
$$

It can be used to show that when $\widetilde{A} \in \mathcal{M}$, and we define

$$
V=\overline{\operatorname{pr}_{\zeta} D(\widetilde{A})}, \quad W=\overline{\operatorname{pr}_{\zeta^{\prime}} D\left(\widetilde{A}^{*}\right)},
$$

then

$$
\left\{\left\{u_{\zeta},(A u)_{W}\right\} \mid u \in D(\widetilde{A})\right\} \text { is a graph, }
$$

defining an operator $T$ from $D(T) \subset V$ to $W$.

Theorem 5.1. ([G68]) There is a 1-1 correspondence between the closed operators $\widetilde{A} \in \mathcal{M}$ and the closed densely defined operators $T: V \rightarrow W$, where $V \subset Z, W \subset Z^{\prime}$ (arbitrary closed subspaces), such that $\widetilde{A}$ corresponds to $T: V \rightarrow W$ if and only if

$$
D(\widetilde{A})=\left\{u \in D\left(A_{\max }\right) \mid \operatorname{pr}_{\zeta} u \in D(T),(A u)_{W}=T \operatorname{pr}_{\zeta} u\right\} .
$$

In this correspondence, $V=\overline{\operatorname{pr}_{\zeta} D(\widetilde{A})}, W=\overline{\operatorname{pr}_{\zeta^{\prime}} D\left(\widetilde{A}^{*}\right)}$, and

- $\widetilde{A}^{*}$ corresponds analogously to $T^{*}: W \rightarrow V$. 
- $\operatorname{ker} \widetilde{A}=\operatorname{ker} T ; \quad \operatorname{ran} \widetilde{A}=\operatorname{ran} T+(H \ominus W)$.

- $\widetilde{A}$ is bijective if and only if $T$ is so, and then

$$
\widetilde{A}^{-1}=A_{\gamma}^{-1}+\mathrm{i}_{V} T^{-1} \operatorname{pr}_{W} .
$$

One also has

$$
D(\widetilde{A})=\left\{u=v+A_{\gamma}^{-1}(T z+f)+z \mid v \in D\left(A_{\min }\right), z \in D(T), f \in Z \ominus W\right\},
$$

where $v, z$ and $f$ are uniquely determined from $u$.

The result builds on the works of Kreln [K47] and Birman [B56] (for selfadjoint operators) and Vishik [V52], and completes the latter: In Vishik's paper, the $\widetilde{A}$ were set in relation to operators over the nullspaces going in the opposite direction of our T's, and the results were focused on those $\widetilde{A}$ 's that have closed range (the so-called normally solvable realizations). Our analysis covered all closed $\widetilde{A}$.

The condition in (8)

$$
(A u)_{W}=T \operatorname{pr}_{\zeta} u
$$

can be viewed as an "abstract boundary condition".

When $\lambda \in \varrho\left(A_{\gamma}\right)$, one can do the same construction for the operators shifted by subtraction of $\lambda$. We denote

$$
Z_{\lambda}=\operatorname{ker}\left(A_{\max }-\lambda\right), \quad Z_{\bar{\lambda}}^{\prime}=\operatorname{ker}\left(A_{\max }^{\prime}-\bar{\lambda}\right),
$$

and have the decompositions (where $\operatorname{pr}_{\gamma}^{\lambda}=\left(A_{\gamma}-\lambda\right)^{-1}\left(A_{\max }-\lambda\right)$ )

$$
D\left(A_{\max }\right)=D\left(A_{\gamma}\right) \dot{+} Z_{\lambda}, \quad u=u_{\gamma}^{\lambda}+u_{\zeta}^{\lambda}=\operatorname{pr}_{\gamma}^{\lambda} u+\operatorname{pr}_{\zeta}^{\lambda} u,
$$

with a similar notation with primes.

Corollary 5.2. Let $\lambda \in \varrho\left(A_{\gamma}\right)$. For the closed $\widetilde{A} \in \mathcal{M}$, there is a 1-1 correspondence

$$
\widetilde{A}-\lambda \longleftrightarrow\left\{\begin{array}{l}
T^{\lambda}: V_{\lambda} \rightarrow W_{\bar{\lambda}}, \text { closed, densely defined } \\
\text { with } V_{\lambda} \subset Z_{\lambda}, W_{\bar{\lambda}} \subset Z_{\bar{\lambda}}^{\prime}, \text { closed subspaces } .
\end{array}\right.
$$

Here $D\left(T^{\lambda}\right)=\operatorname{pr}_{\zeta}^{\lambda} D(\widetilde{A}), V_{\lambda}=\overline{D\left(T^{\lambda}\right)}, W_{\bar{\lambda}}=\overline{\operatorname{pr}_{\zeta^{\prime}}^{\bar{\lambda}} D\left(\widetilde{A}^{*}\right)}$, and $D(\widetilde{A})$ consists of the functions $u \in D\left(A_{\max }\right)$ such that $u_{\zeta}^{\lambda} \in D\left(T^{\lambda}\right)$ and

$$
T^{\lambda} u_{\zeta}^{\lambda}=((A-\lambda) u)_{W_{\bar{\lambda}}} .
$$

Moreover,

- $\operatorname{ker}(\widetilde{A}-\lambda)=\operatorname{ker} T^{\lambda} ; \quad \operatorname{ran}(\widetilde{A}-\lambda)=\operatorname{ran} T^{\lambda}+\left(H \ominus W_{\bar{\lambda}}\right)$.

- $\widetilde{A}-\lambda$ is bijective if and only if $T^{\lambda}$ is so, and when $\lambda \in \varrho(\widetilde{A}) \cap \varrho\left(A_{\gamma}\right)$,

$$
(\widetilde{A}-\lambda)^{-1}=\left(A_{\gamma}-\lambda\right)^{-1}+\mathrm{i}_{V_{\lambda}}\left(T^{\lambda}\right)^{-1} \operatorname{pr}_{W_{\bar{\lambda}}} .
$$

This gives a Krĕ̌n-type resolvent formula for any closed $\widetilde{A} \in \mathcal{M}$ with $\varrho(\widetilde{A}) \cap$ $\varrho\left(A_{\gamma}\right) \neq \emptyset$.

The relation between $T$ and $T^{\lambda}$ was determined in [G74] in the symmetric case, for real $\lambda$, and the proof given there extends immediately to the general situation (as shown in [BGW09]):

For $\lambda \in \varrho\left(A_{\gamma}\right)$, define

$$
E^{\lambda}=I+\lambda\left(A_{\gamma}-\lambda\right)^{-1}, \text { it has the inverse } F^{\lambda}=I-\lambda A_{\gamma}^{-1},
$$

and similarly $E^{\prime \bar{\lambda}}=I+\bar{\lambda}\left(A_{\gamma}^{*}-\bar{\lambda}\right)^{-1}$ has the inverse $F^{\prime \bar{\lambda}}=I-\bar{\lambda}\left(A_{\gamma}^{*}\right)^{-1}$ on $H$. Then $E^{\lambda} F^{\lambda}=F^{\lambda} E^{\lambda}=I, E^{\prime \bar{\lambda}} F^{\prime \bar{\lambda}}=F^{\prime \bar{\lambda}} E^{\prime \bar{\lambda}}=I$ on $H$.

Moreover, $E^{\lambda}$ and $E^{\prime \bar{\lambda}}$ restrict to homeomorphisms

$$
E_{V}^{\lambda}: V \stackrel{\sim}{\rightarrow} V_{\lambda}, \quad E_{W}^{\prime \bar{\lambda}}: W \stackrel{\sim}{\rightarrow} W_{\bar{\lambda}},
$$


with inverses $F_{V}^{\lambda}$ resp. $F_{W}^{\prime \bar{\lambda}}$. In particular, $D\left(T^{\lambda}\right)=E_{V}^{\lambda} D(T)$.

The operator families derived from $E^{\lambda}$ are related to what was called a gammafield in other works from the 1970's and onwards, as a simple special case.

Theorem 5.3. Let $G_{V, W}^{\lambda}=-\operatorname{pr}_{W} \lambda E^{\lambda} \mathrm{i}_{V}$; then

$$
\left(E_{W}^{\prime \bar{\lambda}}\right)^{*} T^{\lambda} E_{V}^{\lambda}=T+G_{V, W}^{\lambda} .
$$

In other words, $T$ and $T^{\lambda}$ are related by the commutative diagram

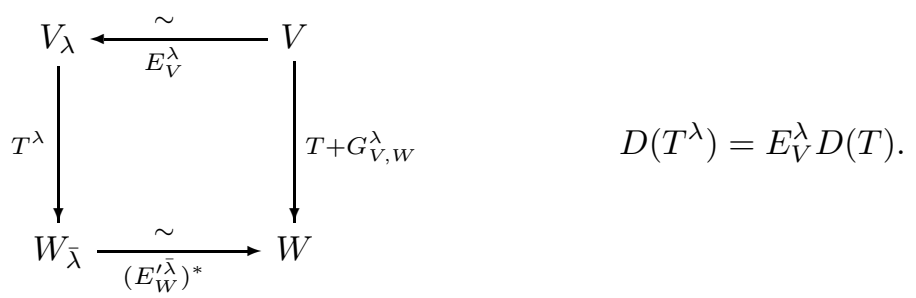

In the joint work with Brown and Wood [BGW09] we moreover showed how the study relates to studies of boundary triples and $M$-functions by other researchers (as referred to in the start af this section; more references are given in [BGW09]):

Let $V=Z, W=Z^{\prime}$, then, with $\mathcal{H}=Z^{\prime}, \mathcal{K}=Z$, the mappings

$$
\begin{aligned}
& \left(\begin{array}{l}
\Gamma_{1} u \\
\Gamma_{0} u
\end{array}\right)=\left(\begin{array}{c}
(A u)_{Z^{\prime}} \\
u_{\zeta}
\end{array}\right): D\left(A_{\max }\right) \rightarrow \mathcal{H} \times \mathcal{K}, \\
& \left(\begin{array}{c}
\Gamma_{1}^{\prime} v \\
\Gamma_{0}^{\prime} v
\end{array}\right)=\left(\begin{array}{c}
\left(A^{\prime} v\right)_{Z} \\
v_{\zeta^{\prime}}
\end{array}\right): D\left(A_{\max }^{\prime}\right) \rightarrow \mathcal{K} \times \mathcal{H},
\end{aligned}
$$

form a boundary triple: Both mappings $\left(\begin{array}{c}\Gamma_{1} \\ \Gamma_{0}\end{array}\right)$ and $\left(\begin{array}{c}\Gamma_{1}^{\prime} \\ \Gamma_{0}^{\prime}\end{array}\right)$ are surjective, their kernels are $D\left(A_{\min }\right)$ resp. $D\left(A_{\min }^{\prime}\right)$, and they satisfy the Green's formula

$$
\left(A_{\max } u, v\right)-\left(u, A_{\max }^{\prime} v\right)=\left(\Gamma_{1} u, \Gamma_{0}^{\prime} v\right)_{\mathcal{H}}-\left(\Gamma_{0} u, \Gamma_{1}^{\prime} v\right)_{\mathcal{K}},
$$

which is a rewriting of (7).

Here one can consider a boundary condition

$$
\Gamma_{1} u=T \Gamma_{0} u,
$$

where we allow $T$ to be unbounded (closed densely defined) from $\mathcal{K}$ to $\mathcal{H}$; it defines a restriction $\widetilde{A}$ of $A_{\max }$ by $D(\widetilde{A})=\left\{u \in D\left(A_{\max }\right) \mid \Gamma_{0} u \in D(T), \Gamma_{1} u=T \Gamma_{0} u\right\}$. Then it is customary to define an $M$-function as follows:

Definition 5.4. For $\lambda \in \varrho(\widetilde{A}), M(\lambda): \operatorname{ran}\left(\Gamma_{1}-T \Gamma_{0}\right) \rightarrow \mathcal{K}$ is the operator satisfying

$$
M(\lambda)\left(\Gamma_{1} x-T \Gamma_{0} x\right)=\Gamma_{0} x, \text { for all } x \in \operatorname{ker}\left(A_{\max }-\lambda\right)=Z_{\lambda} .
$$

The analysis in [BGW09] showed that $M(\lambda)$ is a holomorphic family of operators in $\mathcal{L}(\mathcal{H}, \mathcal{K})$. On the other hand, when $\widetilde{A}$ and its boundary condition (10) are considered from the point of view of extensions [G68]-[G74] recalled further above, $\widetilde{A}$ is the operator corresponding to $T: Z \rightarrow Z^{\prime}$ by Theorem 5.1. Then we find moreover, in terms of the $\lambda$-dependent families introduced in that context:

$$
M(\lambda)=-\left(T+G_{Z, Z^{\prime}}^{\lambda}\right)^{-1}=-F_{Z}^{\lambda}\left(T^{\lambda}\right)^{-1}\left(F_{Z^{\prime}}^{\prime \bar{\lambda}}\right)^{*} \text {, when } \lambda \in \varrho(\widetilde{A}) \cap \varrho\left(A_{\gamma}\right) .
$$

This gives the Kreın resolvent formula in the form

$$
(\widetilde{A}-\lambda)^{-1}=\left(A_{\gamma}-\lambda\right)^{-1}-\mathrm{i}_{Z_{\lambda}} E_{Z}^{\lambda} M(\lambda)\left(E_{Z^{\prime}}^{\prime \bar{\lambda}}\right)^{*} \operatorname{pr}_{Z_{\bar{\lambda}}^{\prime}} .
$$

For the case of general $V$ and $W$, we could likewise construct an $M$-function from $W$ to $V$ for $\lambda \in \varrho(\widetilde{A})$, and establish a Kreln resolvent formula around it. The following result is shown in [BGW09]: 
Theorem 5.5. Let $\widetilde{A}$ correspond to $T: V \rightarrow W$. For $\lambda \in \varrho(\widetilde{A})$ there is a welldefined holomorphic family $M(\lambda) \in \mathcal{L}(W, V)$ :

$$
M(\lambda)=\operatorname{pr}_{\zeta}\left(I-(\widetilde{A}-\lambda)^{-1}\left(A_{\max }-\lambda\right)\right) A_{\gamma}^{-1} \mathrm{i}_{W} .
$$

When $\lambda \in \varrho(\widetilde{A}) \cap \varrho\left(A_{\gamma}\right)$, then

$$
M(\lambda)=-\left(T+G_{V, W}^{\lambda}\right)^{-1}=-F_{V}^{\lambda}\left(T^{\lambda}\right)^{-1}\left(F_{W}^{\prime \bar{\lambda}}\right)^{*},
$$

and

$$
(\widetilde{A}-\lambda)^{-1}=\left(A_{\gamma}-\lambda\right)^{-1}-\mathrm{i}_{V_{\lambda}} E_{V}^{\lambda} M(\lambda)\left(E_{W}^{\prime \bar{\lambda}}\right)^{*} \operatorname{pr}_{W_{\bar{\lambda}}} .
$$

To have both $T^{\lambda}$ (for $\lambda \in \varrho\left(A_{\gamma}\right)$ ) and $M(\lambda)$ (for $\lambda \in \varrho(\widetilde{A})$ ) available is an advantage, since $\operatorname{ker}(\widetilde{A}-\lambda)=\operatorname{ker} T^{\lambda}$ and $\operatorname{ran}(\widetilde{A}-\lambda)=\operatorname{ran} T^{\lambda}+\left(H \ominus W_{\bar{\lambda}}\right)$ give straightforward eigenvalue information at the poles of $M(\lambda)$ in $\varrho\left(A_{\gamma}\right)$.

Remark 5.6. The name $M$-function is consistent with the notation in some papers that [BGW09] refers to, but possibly diverges from others (one could also use the longer name Weyl-Titchmarsh function). There is a recent publication of Malamud [M10] that exposes related resolvent formulas on the basis of [MM02]. (Let us remark that [M10] seemingly ascribes a hypothesis of separate surjectiveness of $\Gamma_{0}$ and $\Gamma_{1}$ to the presentation in [BGW09]; this is not so.)

\section{IMPLEMENTATION OF THE ABSTRACT SET-UP FOR ELLIPTIC OPERATORS}

We shall now recall how the abstract theory is applied to a concrete choice of elliptic operator $A$. Here $A_{\max }$ and $A_{\min }$ are defined as in Section 2; they are closed operators in $H=L_{2}(\Omega)$. In [G68] general even-order operators were considered, and the reference operator (called $A_{\gamma}$ in Section 6) was taken to represent a general normal boundary condition. To simplify our explanation, we shall here just consider a second-order strongly elliptic operator $A$ and let $A_{\gamma}$ stand for the Dirichlet realization, mentioned after (2). We have by elliptic regularity that $D\left(A_{\gamma}\right)=H^{2}(\Omega) \cap H_{0}^{1}(\Omega)$, and we can assume that (a constant has been added to $A$ such that) the lower bound $m\left(A_{\gamma}\right)$ is positive. The lower bound $m(P)$ of a operator $P$ is defined by

$$
m(P)=\inf \{\operatorname{Re}(P u, u) \mid u \in D(P),\|u\|=1\} \geq-\infty ;
$$

when it is finite, $P$ is said to be lower bounded.

The trace operator $\gamma_{0}$ defines a continuous mapping $H^{s}(\Omega) \rightarrow H^{s-\frac{1}{2}}(\Sigma)$ for $s>-\frac{1}{2}$. We shall also need a more advanced fact, namely that, as shown by Lions and Magenes (see e.g. [LM68]), $\gamma_{0}$ extends to a mapping $D\left(A_{\max }\right) \rightarrow H^{-\frac{1}{2}}(\Sigma)$, and defines homeomorphisms

$$
\gamma_{Z}: Z \stackrel{\sim}{\rightarrow} H^{-\frac{1}{2}}(\Sigma), \quad \gamma_{Z^{\prime}}: Z^{\prime} \stackrel{\sim}{\rightarrow} H^{-\frac{1}{2}}(\Sigma),
$$

where $Z$ and $Z^{\prime}$ are the nullspaces of $A_{\max }$ and $A_{\max }^{\prime}$ (not contained in $H^{s}(\Omega)$ for $s>0$ ). The inverse of $\gamma_{Z}$ is consistent with the Poisson operator $K_{\gamma}$ solving the semi-homogeneous Dirichlet problems (2) with $f=0$, in the sense that

$$
K_{\gamma}=\mathrm{i}_{Z} \gamma_{Z}^{-1} \text {. }
$$

Similarly, the inverse $\gamma_{Z^{\prime}}^{-1}$ is consistent with the Poisson solution operator $K_{\gamma}^{\prime}$ solving the Dirichlet problem for $A^{\prime}$ with $f=0, K_{\gamma}^{\prime}=\mathrm{i}_{Z^{\prime}} \gamma_{Z^{\prime}}^{-1}$. Moreover, with $\lambda$ dependence,

$$
K_{\gamma}^{\lambda}=\mathrm{i}_{Z_{\lambda}} \gamma_{Z_{\lambda}}^{-1}, \quad K_{\gamma}^{\prime \bar{\lambda}}=\mathrm{i}_{Z_{\bar{\lambda}}^{\prime}} \gamma_{Z_{\bar{\lambda}}^{\prime}}^{-1}
$$

solve the semi-homogeneous Dirichlet problems for $A-\lambda, A^{\prime}-\bar{\lambda}$, when $\lambda \in \varrho\left(A_{\gamma}\right)$. 
For a closed subspace $V \subset Z$, let $X=\gamma_{0} V \subset H^{-\frac{1}{2}}(\Sigma)$. Here we denote the restriction of $\gamma_{0}$ by $\gamma_{V}$;

$$
\gamma_{V}: V \stackrel{\sim}{\rightarrow} X
$$

with a similar notation for $Y=\gamma_{0} W$ and $\lambda$-dependent cases. The map $\gamma_{V}: V \stackrel{\sim}{\rightarrow} X$ has the adjoint $\gamma_{V}^{*}: X^{*} \stackrel{\sim}{\rightarrow} V$. Here $X^{*}$ denotes the antidual space of $X$, with a duality coinciding with the scalar product in $L_{2}(\Sigma)$ when applied to elements that come from $L_{2}(\Sigma)$. The duality is written $(\psi, \varphi)_{X^{*}, X}$.

We denote

$$
K_{\gamma, X}=\mathrm{i}_{V} \gamma_{V}^{-1}: X \rightarrow V \subset H ;
$$

it is a Poisson operator when $X=H^{-\frac{1}{2}}(\Sigma)$.

Now a given $T: V \rightarrow W$ is carried over to a closed, densely defined operator $L: X \rightarrow Y^{*}$ by the definition

$$
L=\left(\gamma_{W}^{-1}\right)^{*} T \gamma_{V}^{-1}, \quad D(L)=\gamma_{V} D(T) ;
$$

it is expressed in the diagram

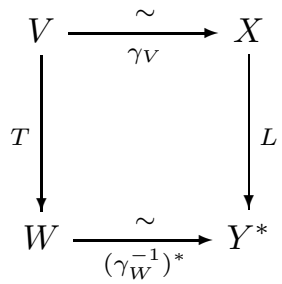

There is a similar definition in the $\lambda$-dependent case.

Before formulating the results in a theorem, we shall interpret the abstract boundary condition (9) defining the realization $\widetilde{A}$, as a concrete condition in terms of $L$.

$A$ has a Green's formula (for sufficiently smooth $u, v$ )

$$
(A u, v)_{\Omega}-\left(u, A^{\prime} v\right)_{\Omega}=\left(\nu_{1} u, \gamma_{0} v\right)_{\Sigma}-\left(\gamma_{0} u, \nu_{1}^{\prime} v\right)_{\Sigma}
$$

where

$$
\nu_{1}=s \gamma_{1}, \quad \nu_{1}^{\prime}=\bar{s} \gamma_{1}+\mathcal{A}^{\prime} \gamma_{0}
$$

with a nonvanishing smooth function $s$ and a suitable first-order differential operator $\mathcal{A}^{\prime}$ on $\Sigma$.

Let $\lambda \in \varrho\left(A_{\gamma}\right)$. In addition to the Poisson operators $K_{\gamma}^{\lambda}$ resp. $K_{\gamma}^{\prime \bar{\lambda}}$ solving the Dirichlet problems for $A-\lambda$ resp. $A^{\prime}-\bar{\lambda}$, we shall need the Dirichlet-to-Neumann operators

$$
P_{\gamma_{0}, \nu_{1}}^{\lambda}=\nu_{1} K_{\gamma}^{\lambda}, \quad P_{\gamma_{0}, \nu_{1}^{\prime}}^{\bar{\lambda}}=\nu_{1}^{\prime} K_{\gamma}^{\prime \bar{\lambda}}
$$

that map the Dirichlet boundary value into the Neumann boundary value for nullsolutions. By the composition rules for $\psi$ dbo's, they are pseudodifferential operators of order 1; moreover, it is known that $P_{\gamma_{0}, \nu_{1}}^{\lambda}$ is elliptic.

Theorem 6.1. Define the reduced Neumann trace operator $\Gamma$ by

$$
\Gamma=\nu_{1}-P_{\gamma_{0}, \nu_{1}}^{0} \gamma_{0}=\nu_{1} A_{\gamma}^{-1} A_{\max }: D\left(A_{\max }\right) \rightarrow H^{\frac{1}{2}}(\Sigma) .
$$

It is continuous and surjective, and vanishes on $Z$. With the analogous definition for $A^{\prime}$ one has the reduced Green's formula:

$$
(A u, v)-\left(u, A^{\prime} v\right)=\left(\Gamma u, \gamma_{0} v\right)_{\frac{1}{2},-\frac{1}{2}}-\left(\gamma_{0} u, \Gamma^{\prime} v\right)_{-\frac{1}{2}, \frac{1}{2}},
$$

valid for all $u \in D\left(A_{\max }\right), v \in D\left(A_{\max }^{\prime}\right)$. In particular,

$$
(A u, w)=\left(\Gamma u, \gamma_{0} w\right)_{\frac{1}{2},-\frac{1}{2}}, \text { when } u \in D\left(A_{\max }\right), w \in Z^{\prime} .
$$


For $\lambda \in \varrho\left(A_{\gamma}\right)$ we similarly define

$$
\begin{aligned}
\Gamma^{\lambda} & =\nu_{1}-P_{\gamma_{0}, \nu_{1}}^{\lambda} \gamma_{0}=\nu_{1}\left(A_{\gamma}-\lambda\right)^{-1}\left(A_{\max }-\lambda\right), \\
\Gamma^{\prime \bar{\lambda}} & =\nu_{1}-P_{\gamma_{0}, \nu_{1}^{\prime}}^{\prime \bar{\lambda}} \gamma_{0}=\nu_{1}^{\prime}\left(A_{\gamma}^{*}-\bar{\lambda}\right)^{-1}\left(A_{\max }^{\prime}-\bar{\lambda}\right),
\end{aligned}
$$

continuous and surjective from $D\left(A_{\max }\right)$ resp. $D\left(A_{\max }^{\prime}\right)$ to $H^{\frac{1}{2}}(\Sigma)$; then there holds a reduced Green's formula

$$
(A u, v)-\left(u, A^{\prime} v\right)=\left(\Gamma^{\lambda} u, \gamma_{0} v\right)_{\frac{1}{2},-\frac{1}{2}}-\left(\gamma_{0} u, \Gamma^{\prime \bar{\lambda}} v\right)_{-\frac{1}{2}, \frac{1}{2}},
$$

for $u \in D\left(A_{\max }\right), v \in D\left(A_{\max }^{*}\right)$.

Here $D\left(A_{\max }\right)$ is provided with the graph-norm.

Now let $\widetilde{A}$ be a closed operator lying between $A_{\min }$ and $A_{\max }$, so $\widetilde{A} \in \mathcal{M}$. The abstract boundary condition (9) for $\widetilde{A}$ may be written:

$$
(A u, w)=\left(T u_{\zeta}, w\right), \text { all } w \in W .
$$

The left-hand side equals $\left(\Gamma u, \gamma_{0} w\right)_{\frac{1}{2},-\frac{1}{2}}$ by (15). The right-hand side equals

$$
\left(T u_{\zeta}, w\right)=\left(T \gamma_{V}^{-1} \gamma_{0} u, \gamma_{W}^{-1} \gamma_{0} w\right)=\left(L \gamma_{0} u, \gamma_{0} w\right)_{Y^{*}, Y}
$$

by definition of $L$ (it is used that when $u_{\zeta} \in V, u_{\zeta}=\gamma_{V}^{-1} \gamma_{V} u_{\zeta}=\gamma_{V}^{-1} \gamma_{0} u$ ).

Hence (16) may be written

$$
\left(\Gamma u, \gamma_{0} w\right)_{\frac{1}{2},-\frac{1}{2}}=\left(L \gamma_{0} u, \gamma_{0} w\right)_{Y^{*}, Y}, \text { all } w \in W .
$$

The injection $\mathrm{i}_{Y}: Y \rightarrow H^{-\frac{1}{2}}(\Sigma)$ has as adjoint the mapping $\mathrm{i}_{Y}^{*}: H^{\frac{1}{2}}(\Sigma) \rightarrow Y^{*}$ that sends a functional $\psi$ on $H^{-\frac{1}{2}}(\Sigma)$ over into a functional $\mathrm{i}_{Y}^{*} \psi$ on $Y$ by:

$$
\left(\mathrm{i}_{Y}^{*} \psi, \varphi\right)_{Y^{*}, Y}=(\psi, \varphi)_{\frac{1}{2},-\frac{1}{2}} \text { for all } \varphi \in Y .
$$

Then (17) may be rewritten as

$$
\mathrm{i}_{Y}^{*} \Gamma u=L \gamma_{0} u
$$

or, when we use that $\Gamma=\nu_{1}-P_{\gamma_{0}, \nu_{1}}^{0} \gamma_{0}$,

$$
\mathrm{i}_{Y}^{*} \nu_{1} u=\left(L+\mathrm{i}_{Y}^{*} P_{\gamma_{0}, \nu_{1}}^{0}\right) \gamma_{0} u \text {. }
$$

This is the boundary condition derived from (9), when $\widetilde{A}$ corresponds to $T: V \rightarrow W$ by Theorem 5.1 , carried over to $L: X \rightarrow Y^{*}$ by (12).

Then Theorem 5.1 implies:

Theorem 6.2. There is a 1-1 correspondence between the closed operators $\widetilde{A} \in \mathcal{M}$ and the closed densely defined operators $L: X \rightarrow Y^{*}$, where $X$ and $Y$ are closed subspaces of $H^{-\frac{1}{2}}(\Sigma)$, such that $\widetilde{A}$ corresponds to $L: X \rightarrow Y^{*}$ if and only if $D(\widetilde{A})$ consists of the functions in $D\left(A_{\max }\right)$ for which

$$
\gamma_{0} u \in D(L), \quad \mathrm{i}_{Y}^{*} \nu_{1} u=\left(L+\mathrm{i}_{Y}^{*} P_{\gamma_{0}, \nu_{1}}^{0}\right) \gamma_{0} u .
$$

In this correspondence, $X=\overline{\gamma_{0} D(\widetilde{A})}, Y=\overline{\gamma_{0} D\left(\widetilde{A}^{*}\right)}$, and

- $\widetilde{A}^{*}$ corresponds analogously to $L^{*}: Y \rightarrow X^{*}$.

- $\operatorname{ker} \widetilde{A}=\mathrm{i}_{V} \gamma_{V}^{-1} \operatorname{ker} L ; \quad \operatorname{ran} \widetilde{A}=\gamma_{W}^{*} \operatorname{ran} L+(H \ominus W)$, cf. (12), (13).

- $\widetilde{A}$ is bijective if and only if $L$ is so, and then

$$
\widetilde{A}^{-1}=A_{\gamma}^{-1}+\mathrm{i}_{V} \gamma_{V}^{-1} L^{-1} \gamma_{W}^{*} \operatorname{pr}_{W}=A_{\gamma}^{-1}+K_{\gamma, X} L^{-1}\left(K_{\gamma, Y}^{\prime}\right)^{*}
$$


Theorems 6.1 and 6.2 are from [G68], except that we have modified the notation a little.

In [BGW09], the subspace cases are treated with insertion of an isometry $\Lambda_{\frac{1}{2}}: L_{2}(\Sigma) \stackrel{\sim}{\rightarrow} H^{-\frac{1}{2}}(\Sigma)$, that allows replacing $X$ and $Y$ by closed subspaces $X_{1}$ and $Y_{1}$ of $L_{2}(\Sigma)$, identified with their dual spaces; then $\mathrm{i}_{Y}^{*}$ is replaced by an orthogonal projection $\operatorname{pr}_{Y_{1}}$.

In the case where $Y=H^{-\frac{1}{2}}(\Sigma)$, i.e., $W=Z^{\prime}$, the map $\mathrm{i}_{Y}^{*}$ is superfluous, and the second condition in (19) takes the form

$$
\nu_{1} u=\left(L+P_{\gamma_{0}, \nu_{1}}^{0}\right) \gamma_{0} u .
$$

When also $X=H^{-\frac{1}{2}}(\Sigma)$, we say that $\widetilde{A}$ represents a Neumann-type condition

$$
\nu_{1} u=C \gamma_{0} u \text {; here } C=L+P_{\gamma_{0}, \nu_{1}}^{0} \text { on } D(L) \text {. }
$$

In this case, $L$ can act like a pseudodifferential operator, namely when $C$ (in the condition $\left.\nu_{1} u=C \gamma_{0} u\right)$ is a differential or pseudodifferential operator.

Let us consider a slightly different set-up where $C$ is a given first-order differential or pseudodifferential operator on $\Sigma$, and we define $\widetilde{A}$ by

$$
D(\widetilde{A})=\left\{u \in D\left(A_{\max }\right) \mid \nu_{1} u=C \gamma_{0} u\right\},
$$

where $\gamma_{0}$ and $\nu_{1}$ are considered as mappings from $D\left(A_{\max }\right)$ to $H^{-\frac{1}{2}}(\Sigma)$ resp. $H^{-\frac{3}{2}}(\Sigma)$. We shall discuss the corresponding operator $L: X \rightarrow Y^{*}$. Since $\left\{\gamma_{0} u, \nu_{1} u\right\}: H^{2}(\Omega) \rightarrow H^{\frac{3}{2}}(\Sigma) \times H^{\frac{1}{2}}(\Sigma), D(L)=\gamma_{0} D(\widetilde{A}) \supset H^{\frac{3}{2}}(\Sigma)$. Then since $H^{\frac{3}{2}}(\Sigma)$ is dense in $H^{-\frac{1}{2}}(\Sigma), X=H^{-\frac{1}{2}}(\Sigma)$. By use of Green's formula (14) it is checked that the adjoint $\widetilde{A}^{*}$ extends the realization of $A^{\prime}$ with domain consisting of the functions $v \in H^{2}(\Omega)$ satisfying

$$
\nu_{1}^{\prime} v=C^{*} \gamma_{0} v,
$$

so also $Y=H^{-\frac{1}{2}}(\Sigma)$. Thus we are in the case of Neumann-type boundary conditions, so by comparison with (6), it is seen that $L$ acts like $C-P_{\gamma_{0}, \nu_{1}}^{0}$.

The domain $D(L)$ equals $\left\{\varphi \in H^{-\frac{1}{2}}(\Sigma) \mid\left(C-P_{\gamma_{0}, \nu_{1}}^{0}\right) \varphi \in H^{\frac{1}{2}}(\Sigma)\right\}$; it may not be easy to determine more exactly. Note that $L$ is used as a map from its domain in $H^{-\frac{1}{2}}(\Sigma)$ to $H^{+\frac{1}{2}}(\Sigma)$, although it acts like a $\psi$ do of order 1 .

One case is clear, though: If $C-P_{\gamma_{0}, \nu_{1}}^{0}$ is elliptic of order 1 , then $L \varphi \in H^{\frac{1}{2}}(\Sigma)$ implies $\varphi \in H^{\frac{3}{2}}(\Sigma)$; in this case $D(L)=H^{\frac{3}{2}}(\Sigma)$. Moreover, $D(\widetilde{A}) \subset H^{2}(\Omega)$. A check of the boundary symbol rules shows that this is precisely the case where the system $\left\{A, \nu_{1}-C \gamma_{0}\right\}$ is elliptic. Here we have:

Theorem 6.3. Let $C$ be a first-order differential or pseudodifferential operator on $\Sigma$ and define the realization $\widetilde{A}$ of $A$ by (22). Then if $C-P_{\gamma_{0}, \nu_{1}}^{0}$ is elliptic, the operator $L: X \rightarrow Y^{*}$ corresponding to $\widetilde{A}$ by Theorem 6.2 acts like $C-P_{\gamma_{0}, \nu_{1}}^{0}$ and has

$$
X=Y=H^{-\frac{1}{2}}(\Sigma), \quad D(L)=H^{\frac{3}{2}}(\Sigma) .
$$

Moreover, $D(\widetilde{A}) \subset H^{2}(\Omega)$. Related statements hold for the adjoint $\widetilde{A}^{*}$.

A Robin condition $\nu_{1} u=b \gamma_{0} u$, with a smooth function $b(x)$ on $\Sigma$, is elliptic, since $L$ acts like $b-P_{\gamma_{0}, \nu_{1}}^{0}$, where $P_{\gamma_{0}, \nu_{1}}^{0}$ is elliptic of order 1 and $b$ is of order 0 .

In the case of Theorem 6.3, when $L$ is bijective, the formula (20) has the form

$$
\widetilde{A}^{-1}=A_{\gamma}^{-1}+K_{\gamma} L^{-1}\left(K_{\gamma}^{\prime}\right)^{*},
$$

where all ingredients belong to the $\psi$ dbo calculus: $K_{\gamma}$ is a Poisson operator, $L^{-1}$ is a $\psi$ do on $\Sigma,\left(K_{\gamma}^{\prime}\right)^{*}$ is a trace operator of class 0 , and the composition $K_{\gamma} L^{-1}\left(K_{\gamma}^{\prime}\right)^{*}$ 
is a singular Green operator (of class 0 ). $A_{\gamma}^{-1}$ is the sum $Q_{+}+G$ of a truncated $\psi$ do $Q$ on $\mathbb{R}^{n}$ and a singular Green operator, as in (4).

Let us include a few words on higher-order cases: When $A$ is of order $2 m$, there is a Green's formula generalizing (14), where $\gamma_{0}$ is replaced by an $m$-vector $\gamma=\left\{\gamma_{0}, \ldots, \gamma_{m-1}\right\}$, and $\nu_{1}$ and $\nu_{1}^{\prime}$ are replaced by $m$-vectors of trace operators of orders $m, \ldots, 2 m-1$, mapping into products of Sobolev spaces of different orders over $\Sigma$. One then gets vector versions of the reduced Neumann trace operators $\Gamma$ and $\Gamma^{\prime}$, with matrix-formed versions of the Dirichlet-to-Neumann pseudodifferential operators, but the basic mechanisms in the interpretation are the same. There are interesting cases of subspaces $X, Y$ of the products of Sobolev spaces over $\Sigma$, where ellipticity considerations are relevant. Details are given in [G68]-[G74] and [BGW09].

\section{RESOlvent FORMULAS}

When $\lambda \in \varrho\left(A_{\gamma}\right)$, there is a similar representation of $\widetilde{A}-\lambda$ in terms of a boundary condition defined from an operator $L^{\lambda}$ acting over the boundary. Here it is of particular interest to find the connection between $L$ and $L^{\lambda}$, just as we found the connection between $T$ and $T^{\lambda}$. It turns out that the relation between $L$ and $L^{\lambda}$ is simpler: They both go from $X$ to $Y^{*}$, whereas $T$ resp. $T^{\lambda}$ map between different spaces due to the shift from $Z$ to $Z_{\lambda}$. This holds, since

$$
D(L)=\gamma_{0} D(\widetilde{A})=\gamma_{0} D(\widetilde{A}-\lambda)=D\left(L^{\lambda}\right), \quad X=\overline{D(L)}=\overline{D\left(L^{\lambda}\right)},
$$

with similar statements for $D\left(L^{*}\right), D\left(\left(L^{\lambda}\right)^{*}\right)$ and $Y$. Then we have:

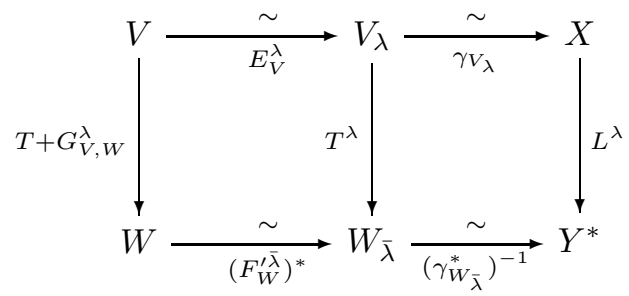

The horizontal maps compose as $\gamma_{V_{\lambda}} E_{V}^{\lambda}=\gamma_{V},\left(\gamma_{W_{\bar{\lambda}}}^{*}\right)^{-1}\left(F_{W}^{\prime \bar{\lambda}}\right)^{*}=\left(\gamma_{W}^{*}\right)^{-1}$, so

$$
L^{\lambda}=\gamma_{V}^{-1}\left(T+G_{V, W}^{\lambda}\right)\left(\gamma_{W}^{*}\right)^{-1} .
$$

In terms of $L^{\lambda}$, the boundary condition reads (analogously to (18)):

$$
\mathrm{i}_{Y}^{*} \nu_{1} u=\left(L^{\lambda}+\mathrm{i}_{Y}^{*} P_{\gamma_{0}, \nu_{1}}^{\lambda}\right) \gamma_{0} u, \gamma_{0} u \in D(L) .
$$

Since $D(\widetilde{A}-\lambda)=D(\widetilde{A})$ is at the same time defined by the boundary condition $\mathrm{i}_{Y}^{*} \nu_{1} u=\left(L+\mathrm{i}_{Y}^{*} P_{\gamma_{0}, \nu_{1}}^{0}\right) \gamma_{0} u$ for $\gamma_{0} u \in D(L)$, we have that $L^{\lambda}+\mathrm{i}_{Y}^{*} P_{\gamma_{0}, \nu_{1}}^{\lambda}=L+\mathrm{i}_{Y}^{*} P_{\gamma_{0}, \nu_{1}}^{0}$ on $D(L)$, so

$$
L^{\lambda}=L+\mathrm{i}_{Y}^{*}\left(P_{\gamma_{0}, \nu_{1}}^{0}-P_{\gamma_{0}, \nu_{1}}^{\lambda}\right) \text { on } D(L) .
$$

The last formula is convenient, since $P_{\gamma_{0}, \nu_{1}}^{0}-P_{\gamma_{0}, \nu_{1}}^{\lambda}$ can be shown to be bounded from $H^{-\frac{1}{2}}(\Sigma)$ to $H^{\frac{1}{2}}(\Sigma)$; hence $L^{\lambda}$ is a perturbation of $L$ by a bounded operator.

Also the general $M$-function defined in Section 5 carries over to an $M$-function on the boundary, a holomorphic family of operators $M_{L}(\lambda) \in \mathcal{L}\left(Y^{*}, X\right)$ defined for $\lambda \in \varrho(\widetilde{A})$.

The results are collected in the following theorem:

Theorem 7.1. Let $\widetilde{A}$ correspond to $T: V \rightarrow W$ as in Theorem 5.1, carried over to $L: X \rightarrow Y^{*}$ as in Theorem 6.2. For $\lambda \in \varrho\left(A_{\gamma}\right)$ it is also described by the boundary condition (24), and there holds: 
(i) For $\lambda \in \varrho\left(A_{\gamma}\right), P_{\gamma_{0}, \nu_{1}}^{0}-P_{\gamma_{0}, \nu_{1}}^{\lambda} \in \mathcal{L}\left(H^{-\frac{1}{2}}(\Sigma), H^{\frac{1}{2}}(\Sigma)\right)$ and

$$
L^{\lambda}=L+\mathrm{i}_{Y}^{*}\left(P_{\gamma_{0}, \nu_{1}}^{0}-P_{\gamma_{0}, \nu_{1}}^{\lambda}\right) \text { on } D(L) .
$$

(ii) For $\lambda \in \varrho(\widetilde{A})$, there is a related $M$-function $\in \mathcal{L}\left(Y^{*}, X\right)$,

$$
M_{L}(\lambda)=\gamma_{0}\left(I-(\widetilde{A}-\lambda)^{-1}\left(A_{\max }-\lambda\right)\right) A_{\gamma}^{-1} \mathrm{i}_{W} \gamma_{W}^{*}
$$

(iii) For $\lambda \in \varrho(\widetilde{A}) \cap \varrho\left(A_{\gamma}\right)$,

$$
M_{L}(\lambda)=-\left(L^{\lambda}\right)^{-1}=-\left(L+\mathrm{i}_{Y}^{*}\left(P_{\gamma_{0}, \nu_{1}}^{0}-P_{\gamma_{0}, \nu_{1}}^{\lambda}\right) \mathrm{i}_{X}\right)^{-1}
$$

and we have the Kreŭn-type resolvent formulas:

$$
(\widetilde{A}-\lambda)^{-1}-\left(A_{\gamma}-\lambda\right)^{-1}=K_{\gamma, X}^{\lambda}\left(L^{\lambda}\right)^{-1}\left(K_{\gamma, Y}^{\prime \bar{\lambda}}\right)^{*}=-K_{\gamma, X}^{\lambda} M_{L}(\lambda)\left(K_{\gamma, Y}^{\prime \bar{\lambda}}\right)^{*}
$$

In the case of a Neumann-type boundary condition as in $(22), L^{\lambda}=C-P_{\gamma_{0}, \nu_{1}}^{\lambda}$ on $D\left(L^{\lambda}\right)=D(L)$.

\section{Applications of pSEUdodifferential methods I: \\ CONDITIONS FOR LOWER BOUNDEDNESS}

The formulas we have shown so far use the terminology of $\psi$ dbo's mainly as a way to indicate what the ingredients in certain operator compositions are. The next question to consider is how properties of $\widetilde{A}$ are reflected in properties of $L$. Part of the analysis can be carried out with methods of functional analysis, but there also exist problems that are solved most efficiently by involving deeper pseudodifferential principles.

An example of how functional analytic and pseudodifferential methods are useful together, is the question of lower boundedness inequalities.

We here restrict the attention to the symmetric set-up where $A$ is formally selfadjoint (so $A_{\max }=A_{\min }^{*}$ ) and $A_{\gamma}$ is selfadjoint; methods for extending the results to nonsymmetric set-ups are found in [G74]. We assume that $A_{\gamma}$ has positive lower bound $m\left(A_{\gamma}\right)$ (cf. (11)).

In the symmetric case, the general extensions $\widetilde{A}$ can of course be nonsymmetric (since $A_{\max }$ is so). Let us speak of the "selfadjoint case" when only selfadjoint $\widetilde{A}$ 's are considered.

In the following, we assume throughout that $\widetilde{A}$ corresponds to $T: V \rightarrow W$ as in Theorem 5.1, and to $L: X \rightarrow Y^{*}$ as in Theorem 6.2. The lower boundedness problem is the problem of how information on lower bounds on $\widetilde{A}$ is related to similar information on $T$ or $L$. The following was shown in [G70] (together with studies of coerciveness estimates):

Theorem 8.1. In the symmetric set-up with $A_{\gamma}$ selfadjoint positive, let $\widetilde{A}$ correspond to $T: V \rightarrow W$ as in Theorem 5.1.

$1^{\circ}$ If $V \subset W$ and $T$ has a lower bound $m(T)$ satisfying $m(T)>-m\left(A_{\gamma}\right)$, then $m(\widetilde{A}) \geq m(T) m\left(A_{\gamma}\right) /\left(m(T)+m\left(A_{\gamma}\right)\right)$.

$2^{\circ}$ Assume that $A_{\gamma}$ is the Friedrichs extension of $A_{\min }$. If $m(\widetilde{A})>-\infty$, then $V \subset W$ and $m(T) \geq m(\widetilde{A})$.

In the selfadjoint case these rules go back to Birman [B56], preceded by sesquilinear form results by Kreı̆n [K47].

The properties of $T$ are easily translated to similar properties of $L$ using the homeomorphism (6); here when $X \subset Y$, we set

$$
m_{-\frac{1}{2}}(L)=\inf \left\{\operatorname{Re}(L \varphi, \varphi)_{Y^{*}, Y} \mid \varphi \in D(L),\|\varphi\|_{-\frac{1}{2}}=1\right\},
$$


for some choice of norm $\|\varphi\|_{-\frac{1}{2}}$ on $H^{-\frac{1}{2}}(\Sigma)$. (One can let $\gamma_{Z}$ be an isometry, to carry numerical information over between $T$ and $L$. Sometimes qualitative objects such as the sign of $m_{-\frac{1}{2}}(L)$ are sufficiently interesting.) To take $A_{\gamma}$ as the Friedrichs extension of $A_{\min }$ means that it is taken as the Dirichlet realization.

In $1^{\circ}$ we see that the statement " $m(T)>-\infty \Longrightarrow m(\widetilde{A})>-\infty$ " holds under the additional assumption that $m(T)>-m\left(A_{\gamma}\right)$; there is a nontrivial question of when that assumption can be removed. In [G74] it was shown that when $A_{\gamma}$ is the Friedrichs extension and $A_{\gamma}^{-1}$ is a compact operator, then $m(T)>-\infty$ does imply $m(\widetilde{A})>-\infty$. This same result was also announced by Mikhailets and Gorbachuk in [GM76] for the selfadjoint case.

In the application to boundary value problems, we therefore have from this early result that lower boundedness of $\widetilde{A}$ and $L$ hold simultaneously when we consider problems on bounded domains $\Omega$, for then $A_{\gamma}^{-1}$ is indeed compact.

For unbounded domains, the question has, to our knowledge, remained unsolved up until recently. The question is closely connected with the comparison of $T$ with $T^{\lambda}$ as in Theorem 5.3. Indeed, as shown in [G74]:

Proposition 8.2. Let $G_{V, W}^{\lambda}$ be as defined in Theorem 5.3. The property

$$
m\left(G_{Z, Z}^{\mu}\right) \rightarrow \infty \text { for } \mu \rightarrow-\infty, \mu \in \mathbb{R},
$$

is necessary and sufficient for the validity of

$$
m(T)>-\infty \Longrightarrow m(\widetilde{A})>-\infty
$$

for general closed $\widetilde{A} \in \mathcal{M}$.

The question was also studied later by Derkach and Malamud [DM91] who worked out an analysis that generalizes Proposition 8.2 and gives further conditions for the validity of the conclusion from $m(T)$ to $m(\widetilde{A})$. However this did not capture elliptic problems on unbounded domains $(n \geq 2)$.

Because of the recent interest in the analysis of extensions, we have considered the problem again, and found a solution in [G11a] for exterior domains (complements in $\mathbb{R}^{n}$ of bounded domains).

Theorem 8.3. Let $\Omega$ be the complement of a smooth bounded set $\bar{\Omega}_{-}$in $\mathbb{R}^{n}$, and let $A$ be symmetric and uniformly strongly elliptic on $\Omega$ with coefficients in $C_{b}^{\infty}(\bar{\Omega})$, and with a positive lower bound for $A_{\gamma}$. In the application of the extension theory to this situation, (27) holds, and hence also (28).

Here $C_{b}^{\infty}(\bar{\Omega})$ stands for the $C^{\infty}$-functions that are bounded with bounded derivatives.

The proof relies on the "translation" of abstract operators $\widetilde{A}$ to concrete operators defined by boundary conditions. Indeed, it turns out that the lower bound of $G_{Z, Z}^{\mu}$ behaves like the lower bound $m_{-\frac{1}{2}}\left(Q^{\mu}\right)$ (cf. (26)) of $Q^{\mu}=P_{\gamma_{0}, \nu_{1}}^{0}-P_{\gamma_{0}, \nu_{1}}^{\mu}$. Then the deep part of the proof lies in setting the operator $Q^{\mu}$ in relation to the analogous operator for the interior domain $\Omega_{-}, Q_{-}^{\mu}$, which does have the desired property, in view of our knowledge of problems on bounded domains. The point is to show that $\left|\left(\left(Q^{\mu}-Q_{-}^{\mu}\right) \varphi, \varphi\right)\right|$ is bounded by $c\|\varphi\|_{L_{2}(\Sigma)}^{2}$ uniformly for $\mu \rightarrow-\infty$, so that addition of $Q^{\mu}-Q_{-}^{\mu}$ to $Q_{-}^{\mu}$ does not violate the growth of the lower bound. This goes by a delicate application of the $\psi$ dbo calculus. Details are in [G11a].

Let us mention that there is a considerably easier result that holds regardless of boundedness of $\partial \Omega$ and only requires some uniformity in the estimates of coefficients of the operators, namely preservation of coerciveness inequalities (Gårding inequalities). We here assume that $\Omega$ is a subset of $\mathbb{R}^{n}$ with smooth boundary, admissible 
as defined in [G96] (besides bounded domains, this allows exterior domains, perturbed halfspaces and other cases that can be covered with a finite system of local coordinates of a relatively simple kind). Moreover, we assume that $A$ is uniformly strongly elliptic on $\bar{\Omega}$ with coefficients in $C_{b}^{\infty}(\bar{\Omega})$. The result is that then $\widetilde{A}$ satisfies a Gårding inequality (with $c>0, k \in \mathbb{R}$ )

$$
\operatorname{Re}(\widetilde{A} u, u) \geq c\|u\|_{1}^{2}-k\|u\|_{0}^{2}, \quad u \in D(\widetilde{A}),
$$

if and only if $X \subset Y$ and $L$ satisfies an inequality

$$
\operatorname{Re}(L \varphi, \varphi) \geq c^{\prime}\|\varphi\|_{H^{\frac{1}{2}}(\Sigma)}^{2}-k^{\prime}\|\varphi\|_{H^{-\frac{1}{2}}(\Sigma)}^{2}, \quad \varphi \in D(L) .
$$

In the case of differential or pseudodifferential Neumann-type boundary conditions, the inequality (29) for $L$ holds precisely when the pseudodifferential operator it acts like, is strongly elliptic. Details for bounded sets are in [G70] for realizations of $2 m$-order operators; the extension to unbounded sets is shown in [G11a] - the argumentation just involves standard trace theorems and interpolation inequalities.

\section{Applications of PSEUdodifferential methods II: SPECTRAL ASYmptotics}

In the symmetric set-up, when $\Omega$ is bounded, the eigenvalues of the selfadjoint operator $A_{\gamma}$ form a sequence $\lambda_{j}$ going to $\infty$ on $\mathbb{R}$. Already in 1912, Weyl [W12] showed the famous estimate for $A=-\Delta$, for $n=2,3, m=2$ :

$$
\lambda_{j}\left(A_{\gamma}\right)-c_{0} j^{m / n} \text { is } o\left(j^{m / n}\right) \text { for } j \rightarrow \infty,
$$

where $c_{0}$ is a constant defined from the volume of $\Omega$; the eigenvalues are repeated according to multiplicities. Equivalently, the counting function $N\left(t ; A_{\gamma}\right)$ (counting the number of eigenvalues in $[0, t])$, and the eigenvalues $\mu_{j}$ of the inverse $A_{\gamma}^{-1}$, satisfy

where

$$
\begin{aligned}
& N\left(t ; A_{\gamma}\right)-c_{A} t^{n / m} \text { is } o\left(t^{n / m}\right) \text { for } t \rightarrow \infty, \\
& \mu_{j}\left(A_{\gamma}^{-1}\right)-c_{A}^{m / n} j^{-m / n} \text { is } o\left(j^{-m / n}\right) \text { for } j \rightarrow \infty,
\end{aligned}
$$

$$
c_{A}=\int_{x \in \Omega, a^{0}(x, \xi)<1} d x d \xi
$$

(and $c_{0}=c_{A}^{-m / n}$ ). The estimates have been shown for general $n$ and sharpened since then, with more precision on the remainder, and the validity has been extended to general elliptic operators $A$ and boundary conditions, and to elliptic pseudodifferential operators $P$ on compact manifolds. These improvements have a long history that we shall not try to account for here in detail; they are interesting not only because of the results but even more because of the refined theories that were invented in connection with the proofs (for example: Fourier integral operators). See e.g. Hörmander [H68], [H71]. Brüning [B74], Seeley [Se78], Ivrii [I82, I84, I91], Safarov and Vassiliev [SV97].

We shall here be concerned with a slightly different question, namely the spectral behavior of the difference between the resolvents of two realizations of $A$.

Birman showed in [B62] for second-order symmetric uniformly strongly elliptic operators $A$ that the singular numbers $s_{j}(B)=\mu_{j}\left(B^{*} B\right)^{\frac{1}{2}}$ of the compact operator $B=\widetilde{A}^{-1}-A_{\gamma}^{-1}$ satisfy an upper estimate:

$$
s_{j}\left(\widetilde{A}^{-1}-A_{\gamma}^{-1}\right) \leq C j^{-2 /(n-1)}, \text { for all } j,
$$

when $\widetilde{A}$ is a selfadjoint realization of $A$ defined by a Neumann or Robin condition. In other words, $\widetilde{A}^{-1}-A_{\gamma}^{-1}$ belongs $\mathfrak{S}_{((n-1) / 2)}$, where $\mathfrak{S}_{(p)}$ is the space of compact operators $B$ for which $s_{j}(B)$ is $O\left(j^{-\frac{1}{p}}\right)$ (often called a weak Schatten class). It is 
particularly interesting that Birman showed this not just for interior, but also for exterior domains, and under low smoothness assumptions.

We note in passing that the estimate (31) implies that

$$
\widetilde{A}^{-1}-A_{\gamma}^{-1} \in \mathcal{C}_{p} \text { for } p>(n-1) / 2,
$$

where $\mathcal{C}_{p}$ is the $p$-th Schatten class (consisting of the operators $B$ such that $\left.\left(s_{j}(B)\right)_{j \in \mathbb{N}} \in \ell_{p}(\mathbb{N})\right)$; but this is less informative that (31).

One of the fundamental ingredients in these studies is embedding properties, more precisely the knowledge that an operator $B$ that is continuous from $L^{2}(\Omega)$ to $H^{s}(\Omega)$ for some $s>0$ ( $\Omega$ bounded smooth $\left.\subset \mathbb{R}^{n}\right)$ is compact in $L_{2}(\Omega)$ and belongs to $\mathfrak{S}_{(n / s)}$. However, this alone only gives upper bounds on the behavior of singular numbers. To get Weyl-type limit properties one must know more about the differential or pseudodifferential structure of the operators.

The estimate (31) was sharpened to a Weyl-type asymptotic estimate in joint works of Birman and Solomiak 1978-80, see in particular [BS80] which showed a general principle for the spectrum of a ratio of two quadratic forms, implying that

$$
s_{j}\left(\widetilde{A}^{-1}-A_{\gamma}^{-1}\right) j^{2 /(n-1)} \rightarrow c, \text { for } j \rightarrow \infty,
$$

for interior and exterior smooth domains.

Prior to this, a far-reaching result had been shown in [G74], Section 8: We consider a symmetric, strongly elliptic $2 m$-order operator acting in an $N$-dimensional vector bundle $E$ over a smooth compact Riemanninan manifold $\bar{\Omega}$ with boundary $\Sigma$, assuming that the Dirichlet realization $A_{\gamma}$ is invertible. Let $A_{B}$ be a selfadjoint invertible realization defined by a normal boundary condition

$$
\sum_{k \leq j} B_{j k} \gamma_{k} u=0, j=0,1, \ldots, 2 m-1,
$$

where the $B_{j k}$ are differential operators of order $j-k$ from $\left.E\right|_{\Sigma}$ to given vector bundles $F_{j}$ over $\Sigma$ (with $\operatorname{dim} F_{j} \geq 0$ ); normality of the boundary condition means that the $B_{j j}$ are surjective morphisms. (That $A$ acts in the vectorbundle $E$ means that it is locally $(N \times N)$-matrix-formed. In the scalar case, $N=1$ and the $F_{j}$ are 0- or 1-dimensional, with $B_{j j}$ an invertible function when $\operatorname{dim} F_{j}=1$. Ellipticity of the boundary condition requires in particular that $\sum_{j} \operatorname{dim} F_{j}=m N$.) Denote $\oplus_{j>m} F_{j}=F^{1}$.

Theorem 9.1. Let $T: V \rightarrow V$ be the operator corresponding to $A_{B}$ by Theorem 5.1. There exists an isometry $J: L_{2}\left(\Sigma, F^{1}\right) \stackrel{\sim}{\rightarrow} V$ with inverse $J^{-1}=J^{*}$ (in the $\psi d$ bo calculus), such that

$$
\mathcal{T}_{1}=J^{*} T J
$$

acts like an elliptic invertible $\psi$ do $\mathcal{T}$ in $F^{1}$ of order $2 m$, and $D\left(\mathcal{T}_{1}\right)=$ $\left\{\varphi \in L_{2}\left(\Sigma, F^{1}\right) \mid \mathcal{T} \varphi \in L_{2}\left(\Sigma, F^{1}\right)\right\}=H^{2 m}\left(\Sigma, F^{1}\right)$. Here $\mathcal{T}_{1}$ has the same spectrum as $T$, and its eigenvectors are mapped to the corresponding eigenvectors of $\mathcal{T}_{1}$ by the isometry $J$. Moreover,

$$
A_{B}^{-1}-A_{\gamma}^{-1}=\mathrm{i}_{V} J \mathcal{T}_{1}^{-1} J^{*} \operatorname{pr}_{V}
$$

whereby the positive resp. negative eigenvalues satisfy

$$
\mu_{j}^{ \pm}\left(A_{B}^{-1}-A_{\gamma}^{-1}\right)=\mu_{j}^{ \pm}\left(\mathcal{T}_{1}^{-1}\right) \text { for all } j .
$$

It follows that with constants determined from the principal symbols,

$$
\begin{aligned}
N^{\prime \pm}\left(t ; A_{B}^{-1}-A_{\gamma}^{-1}\right) & =C^{ \pm} t^{(n-1) /(2 m)}+O\left(t^{(n-1) /(2 m)}\right) \text { for } t \rightarrow \infty, \\
\mu_{j}^{ \pm}\left(A_{B}^{-1}-A_{\gamma}^{-1}\right) & =\left(C^{ \pm}\right)^{2 m /(n-1)} j^{-2 m /(n-1)}+O\left(j^{-(2 m+1) /(n-1)}\right) ;
\end{aligned}
$$

here $N^{\prime \pm}(t ; S)$ indicates the number of positive, resp. negative eigenvalues of $S$ outside the interval $]-1 / t, 1 / t[$. 
The two statements in (36) are equivalent, cf. e.g. [G96], Lemma A.5. They follow from Hörmander [H68] for elliptic $\psi$ do's, when the principal symbol eigenvalues of $\mathcal{T}$ are simple; this restriction is removed by results of Ivrii [I82]. See also Theorem 10.1 below.

We have recently checked that the proof extends to exterior domains, for uniformly strongly elliptic systems.

The fine estimates with remainders depend on the ellipticity of the $\psi$ do. For simple Weyl-type estimates, Birman and Solomiak in [BS77] removed the ellipticity hypothesis, showing that

$$
s_{j}(P) j^{k / n} \rightarrow c\left(p^{0}\right) \text { for } j \rightarrow \infty
$$

holds for any classical $\psi$ do $P$ of order $-k<0$ on a compact manifold of dimension $n$. They even allowed a certain nonsmoothness of the homogeneous principal symbol, both in $x$ and $\xi$, needing only a little more than continuity. In the elliptic case, there are recent works of Ivrii dealing with remainder estimates under weak smoothness hypotheses.

In [G84] we made an effort to increase the accessibility of the $\psi$ dbo calculus by publishing an introduction to it with several improvements, and showing as a main result that any singular Green operator of negative order and class 0 has a Weyl-type spectral asymptotics formula:

Theorem 9.2. Let $G$ be a classical singular Green operator of order $-k<0$ and class 0 on an n-dimensional compact manifold with boundary. It has a spectral asymptotics behavior

$$
s_{j}(G) j^{k /(n-1)} \rightarrow c\left(g^{0}\right) \text { for } j \rightarrow \infty,
$$

where $c\left(g^{0}\right)$ is a constant defined from on the principal symbol of $G$.

This was moreover used to show asymptotic formulas generalizing (33), both for interior and exterior domains, followed up in another study [G84a] including also the dependence on a spectral parameter $\lambda$. Indeed, we have as an immediate corollary of Theorem 9.2, also for nonselfadjoint cases:

Corollary 9.3. Let $A$ be elliptic of order $2 m$ with invertible Dirichlet realization and let $A_{B}$ be an invertible realization defined by a normal elliptic boundary condition. For any positive integer $N, A_{B}^{-N}-A_{\gamma}^{-N}$ is a singular Green operator of order $-2 m N$ and class 0 , and hence satisfies

$$
s_{j}\left(A_{B}^{-N}-A_{\gamma}^{-N}\right) j^{2 m N /(n-1)} \rightarrow c_{N} \text { for } j \rightarrow \infty,
$$

for a constant $c_{N}$ defined from the principal symbols.

Also exterior domains are considered in [G84], [G84a], where (38) is shown for realizations of second-order operators and their iterates. The results apply of course to resolvents by replacement of $A$ by $A-\lambda$; the $\lambda$-dependence is studied in [G84a]. It is seen that the $\psi$ dbo theory provides a forceful tool for such questions, and we strongly recommend its use.

\section{NeW SPECTRAL ReSUlts}

Spectral estimates of resolvent differences have been taken up in recent papers by Behrndt et al. [AB09, BLLLP10, BLL11] for second-order operators and Malamud [M10] for $2 m$-order operators with normal boundary conditions, based on boundary triples methods. Here Schatten class and weak Schatten class estimates are shown, relying on such estimates for Sobolev space embeddings.

We have returned to the subject in [G11] where we, besides showing new results on perturbations of essential spectra, have reformulated and extended results in 
[G84] on estimates like (38), including general differences and exterior domains. The central ingredient is the estimate (37) for singular Green operators, plus the fact that s.g.o.s give their essential contribution in a small neighborhood of the boundary, also for exterior domains, allowing cutoffs eliminating infinity.

An inspection of the results of [G74] shows that the spectral estimates in Theorem 9.1 can be further sharpened by use of results of Ivrii [I82]:

Theorem 10.1. In the setting of Theorem 9.1, assume in addition that the principal symbol of $\mathcal{T}$ satifies Ivrii's conditions $\left(\mathrm{H}_{ \pm}\right)$from [I82] (the bicharateristics through points of $T^{*}(\Sigma) \backslash 0$ are nonperiodic except for a set of measure zero). Then there are constants $C_{1}^{ \pm}$such that

$$
N^{\prime \pm}\left(t ; A_{B}^{-1}-A_{\gamma}^{-1}\right)=C^{ \pm} t^{(n-1) /(2 m)}+C_{1}^{ \pm} t^{(n-2) /(2 m)}+o\left(t^{(n-2) /(2 m)}\right) .
$$

The proof is a direct application of [I82] Th. 0.2 to $\mathcal{T}$.

The formula (35) is a special type of Kreı̆n resolvent formula with isometries, valid for selfadjoint realizations, but the analysis in [G74] also implies Kreln formulas in the nonselfadjoint cases. Namely, Th. 6.4 there shows how $T: V \rightarrow W$ is represented by a realization $\mathcal{L}_{1}$ of a $\psi$ do $\mathcal{L}$ acting between vector bundles over $\Sigma$, and here $A_{B}$ is elliptic if and only if $\mathcal{L}$ is elliptic (Cor. 6.10). In the invertible elliptic case, formula (20) then takes the form

$$
A_{B}^{-1}-A_{\gamma}^{-1}=\mathrm{i}_{V} \gamma_{V}^{-1} \Phi \mathcal{L}_{1}^{-1} \Psi^{*}\left(\gamma_{W}^{*}\right)^{-1} \operatorname{pr}_{W},
$$

with $\mathcal{L}_{1}$ acting like $-\left(B^{10}+B^{11} P_{\gamma, \chi}\right) \Phi$ (notation explained in [G74]); the righthand side is a composition of a Poisson operator, an elliptic $\psi$ do and the adjoint of a Poisson operator, all of mixed order. Its $s$-numbers can be studied by reduction to an elliptic $\psi$ do over $\Sigma$, where Ivrii's sharp results can be used.

Let us just demonstrate this for second-order operators, for the formula (23), with $L^{-1}$ elliptic of order -1 : Denote $\widetilde{A}^{-1}-A_{\gamma}^{-1}=S$. Then

$$
\begin{aligned}
s_{j}(S)^{2} & =s_{j}\left(K_{\gamma} L^{-1}\left(K_{\gamma}^{\prime}\right)^{*}\right)^{2}=\mu_{j}\left(K_{\gamma} L^{-1}\left(K_{\gamma}^{\prime}\right)^{*} K_{\gamma}^{\prime}\left(L^{-1}\right)^{*} K_{\gamma}^{*}\right) \\
& =\mu_{j}\left(L^{-1}\left(K_{\gamma}^{\prime}\right)^{*} K_{\gamma}^{\prime}\left(L^{-1}\right)^{*} K_{\gamma}^{*} K_{\gamma}\right),
\end{aligned}
$$

where we used the general rule $\mu_{j}\left(B_{1} B_{2}\right)=\mu_{j}\left(B_{2} B_{1}\right)$. Both operators $P_{1}=K_{\gamma}^{*} K_{\gamma}$ and $P_{1}^{\prime}=\left(K_{\gamma}^{\prime}\right)^{*} K_{\gamma}^{\prime}$ are selfadjoint positive elliptic $\psi$ do's of order -1 (cf. e.g. [G11b], proof of Th. 4.4). Let $P_{2}=P_{1}^{\frac{1}{2}}$, then we continue the calculations as follows:

$$
s_{j}(S)^{2}=\mu_{j}\left(L^{-1} P_{1}^{\prime}\left(L^{-1}\right)^{*} P_{1}\right)=\mu_{j}\left(P_{2} L^{-1} P_{1}^{\prime}\left(L^{-1}\right)^{*} P_{2}\right)=\mu_{j}\left(P_{3}\right),
$$

where $P_{3}=P_{2} L^{-1} P_{1}^{\prime}\left(L^{-1}\right)^{*} P_{2}$ is a selfadjoint positive elliptic $\psi$ do on $\Sigma$ of order -4. Applying Ivrii's theorem to $P_{3}^{-1}$, we conclude:

Theorem 10.2. For the operator considered in Theorem 6.3, the s-numbers satisfy

$$
N^{\prime}\left(t ; \widetilde{A}^{-1}-A_{\gamma}^{-1}\right)=C t^{(n-1) / 2}+O\left(t^{(n-2) / 2}\right) .
$$

Moreover, if the principal symbol of $P_{3}^{-1}$ satifies Ivrii's condition from [I82] (the bicharateristics through points of $T^{*}(\Sigma) \backslash 0$ are nonperiodic except for a set of measure zero), there is a constant $C_{1}$ such that

$$
N^{\prime}\left(t ; \widetilde{A}^{-1}-A_{\gamma}^{-1}\right)=C t^{(n-1) / 2}+C_{1} t^{(n-2) / 2}+o\left(t^{(n-2) / 2}\right) .
$$

Sharpened asymptotic formulas can also be obtained for differences between resolvents of two realizations that both differ from the Dirichlet realization, by use of the analysis in [G68] with a general invertible realization $A_{\beta}$ as reference operator.

To give another example of applications of the $\psi$ dbo theory, the following result is found straightforwardly as a consequence of [G84]: 
Theorem 10.3. Let $A_{B}$ and $A_{B^{\prime}}$ be elliptic invertible realizations of $A$ such that $B$ and $B^{\prime}$ map into the same bundles and have the same principal part. Then $A_{B}^{-1}-A_{B^{\prime}}^{-1}$ is a singular Green operator of order $-2 m-1$ (since its principal part is zero), and hence, by (37),

$$
s_{j}\left(A_{B}^{-1}-A_{B^{\prime}}^{-1}\right) j^{(2 m+1) /(n-1)} \rightarrow c \text { for } j \rightarrow \infty .
$$

The singular Green operator will be of a still lower order $-2 m-r$ if the first $r>1$ terms in the symbols of $B$ and $B^{\prime}$ coincide.

Example 10.4. As a special case, we can compare two Robin conditions for a second-order operator $A$ :

$$
\begin{aligned}
& \widetilde{A}_{1} \text { defined by } \nu_{1} u=b_{1} \gamma_{0} u, \\
& \widetilde{A}_{2} \text { defined by } \nu_{1} u=b_{2} \gamma_{0} u ;
\end{aligned}
$$

$b_{1}, b_{2} \in C^{\infty}(\Sigma)$. When regarded from the point of view of Theorem 10.3, these are normal boundary conditions $\nu_{1} u-B_{i} \gamma_{0} u=0$, where $B_{1}=b_{1}$ and $B_{2}=b_{2}$ considered as first-order operators have principal part 0 , so the boundary operators have the same principal part. Then the s.g.o. $\widetilde{A}_{1}^{-1}-\widetilde{A}_{2}^{-1}$ is of order -3 , and by $(37)$,

$$
s_{j}\left(\widetilde{A}_{1}^{-1}-\widetilde{A}_{2}^{-1}\right) j^{3 /(n-1)} \rightarrow c \text { for } j \rightarrow \infty .
$$

In [BLLLP10], Berndt, Langer, Lobanov, Lotoreichik and Popov prove upper estimates for this difference in the case $A=-\Delta-\lambda$, namely Schatten class estimates of $s_{j}\left(\widetilde{A}_{1}^{-1}-\widetilde{A}_{2}^{-1}\right)$ as in $(32)$ with $(n-1) / 2$ replaced by $(n-1) / 3$.

In case the $b_{i}$ are $C^{\infty}$, the result is covered by (42) as explained above. However, the $b_{i}$ in [BLLLP10] are allowed to be nonsmooth, namely to be in $L_{\infty}(\Sigma)$, which goes outside the range covered by the smooth $\psi$ dbo theory.

This led us to investigate how far we could push the proof of asymptotic estimates (42) to make them valid for nonsmooth choices of $b_{i}$. The outcome is published in [G11b], where it is shown that (42) holds for symmetric second-order strongly elliptic operators on smooth domains, when $b_{1}$ and $b_{2}$ are piecewise $C^{\varepsilon}$ on $\Sigma$, having jumps at a smooth hypersurface.

The Schatten class estimates have been followed up by Berndt, Langer and Lotoreichik in a study of selfadjoint realizations [BLL11].

Unsolved questions of asymptotic estimates lie primarily in the range of situations with limited smoothness. Resolvent formulas have been studied in such general cases, [PR09] and [G08] for $C^{1,1}$-domains, [GM08, GM11] for Lipschitz and quasi-convex domains, [AGW11] for a class of domains containing $C^{3 / 2+\varepsilon}$, with a nonsmooth generalization of $\psi$ dbo's. To our knowledge, spectral asymptotic estimates have not yet been worked out for such resolvent differences. Some upper estimates are in selfadjoint cases known from Birman [B62].

A problem with a different flavor is the case of a mixed boundary condition, such as prescribing for $-\Delta$ the Dirichlet condition on a part $\Sigma_{-}$of the boundary and a Neumann-type condition on the other part $\Sigma_{+}$. Here there is a jump in the order of the boundary condition. The domain of the realization is contained in $H^{\frac{3}{2}-\varepsilon}(\Omega)$ only for $\varepsilon>0$, so it is not covered by boundary triples methods requiring the domain to be in $H^{\frac{3}{2}}(\Omega)$. Spectral upper estimates are known from [B62]. A spectral asymptotic estimate was obtained recently in [G11c], based on somewhat intricate applications of results on nonstandard pseudodifferential operators.

There are also other questions that can benefit from pseudodifferential methods, for example the study of spectral asymptotics of the nonelliptic Kreln-like extensions, cf. [G11a]. 


\section{REFERENCES}

[AGW11] H. Abels, G. Grubb and I. Wood. Extension theory and KreĬn-type resolvent formulas for nonsmooth boundary value problems, arXiv:1008.3281, to appear.

[A65] S. Agmon. Lectures on Elliptic Boundary Value Problems. Van Nostrand Math. Studies, D. Van Nostrand Publ. Co., Princeton 1965.

[ADN59] S. Agmon, A. Douglis and L. Nirenberg. 1964. Estimates near the boundary for solutions of elliptic partial differential equations satisfying general boundary conditions, I. Comm. Pure Appl. Math, 12, 623-727.

[AB09] D. Alpay and J. Behrndt. 2009. Generalized Q-functions and Dirichlet-to-Neumann maps for elliptic differential operators. J. Funct. Anal. 257, 1666-1694.

[AP04] W. O. Amrein and D. B. Pearson. 2004. $M$ operators: a generalisation of Weyl-Titchmarsh theory. J. Comp. Appl. Math. 171, 1-26.

[A99] Yu. M. Arlinskii. 1999. On functions connected with sectorial operators and their extensions. Integral Equations Operator Theory 33, 125-152.

[BL07] J. Behrndt and M. Langer. 2007. Boundary value problems for elliptic partial differential operators on bounded domains. J. Funct. Anal. 243, 536-565.

[BLLLP10] J. Behrndt, M. Langer, I. Lobanov, V. Lotoreichik and I. Popov. 2010. A remark on Schatten-von Neumann properties of resolvent differences of generalized Robin Laplacians on bounded domains. J. Math. Anal. Appl. 371, 750-758.

[BLL11] J. Behrndt, M. Langer and Lotoreichik. Spectral estimates for differences of resolvents of selfadjoint elliptic operators. arXiv:1012.4596, to appear.

[B56] M. S. Birman. 1956. On the theory of self-adjoint extensions of positive definite operators. Mat. Sb. N.S. 38(80), 431-450. (In Russian.)

[B62] M. S. Birman. 1962. Perturbations of the continuous spectrum of a singular elliptic operator by varying the boundary and the boundary conditions. Vestnik Leningrad. Univ. 17, 22-55. English translation in Spectral theory of differential operators, Amer. Math. Soc. Transl. Ser. 2, 225. Amer. Math. Soc. Providence, R.I. 2008, 19-53.

[BS77] M. S. Birman and M. Z. Solomyak. 1977. Asymptotic behavior of the spectrum of pseudodifferential operators with anisotropically homogeneous symbols. Vestnik Leningrad. Univ. 13, 13-21. English translation in Vestn. Leningr. Univ. Math. 10 (1982), 237-247.

[BS80] M. S. Birman and M. Z. Solomyak. 1980. Asymptotics of the spectrum of variational problems on solutions of elliptic equations in unbounded domains. Funkts. Analiz Prilozhen. 14, 27-35. English translation in Funct. Anal. Appl. 14 (1981), 267-274.

[B71] L. Boutet de Monvel. 1971. Boundary problems for pseudodifferential operators, Acta Math. 126, $11-51$.

[BGW09] B. M. Brown, G. Grubb, and I. G. Wood. 2009. $M$-functions for closed extensions of adjoint pairs of operators with applications to elliptic boundary problems. Math. Nachr. 282, 314-347.

[BMNW08] B. M. Brown, M. Marletta, S. Naboko and I. G. Wood. 2008. Boundary triplets and M-functions for non-selfadjoint operators, with applications to elliptic PDEs and block operator matrices. J. Lond. Math. Soc. 77, 700-718.

[B74] J. Brüning. 1974. Zur Abschätzung der Spektralfunktion elliptischer Operatoren. Math. Z. 137, $75-85$.

[BGP06] J. Brüning, V. Geyler and K. Pankrashkin. 2008. Spectra of self-adjoint extensions and applications to solvable Schrödinger operators. Rev. Math. Phys. 20, 1-70.

[CZ57] A.-P. Calderón and A. Zygmund. 1957. Singular integral operators and differential equations. Amer. J. Math. 79, 901-921.

[CH53] R. Courant and D. Hilbert. Methods of Mathematical Physics, Vol. I. Interscience Publishers, Inc., New York, N.Y. 1953.

[CH62] R. Courant and D. Hilbert. Methods of Mathematical Physics, Vol. II: Partial Differential Equations (by R. Courant). Interscience Publishers (a division of John Wiley \& Sons), New York-London 1962.

[DM91] V. A. Derkach and M. M. Malamud. 1991. Generalized resolvents and the boundary value problems for Hermitian operators with gaps. J. Funct. Anal. 95, 1-95.

[F34] K. Friedrichs. 1934. Spektraltheorie halbbeschränkter Operatoren und Anwendung auf die Spektralzerlegung von Differentialoperatoren. Math. Ann. 109, 465-487.

[GM08] F. Gesztesy and M. Mitrea. 2008. Generalized Robin boundary conditions, Robin-toDirichlet maps, and Krein-type resolvent formulas for Schrödinger operators on bounded Lipschitz domains. Perspectives in Partial Differential Equations, Harmonic Analysis and Applications: A Volume in Honor of Vladimir G. Maz'ya's 70th Birthday, Proceedings of Symposia in Pure Mathematics (eds. D. Mitrea and M. Mitrea) 79, Amer. Math. Soc., Providence, R.I., 105-173. 
[GM11] F. Gesztesy and M. Mitrea. 2011. A description of all selfadjoint extensions of the Laplacian and Krel̆n-type resolvent formulas in nonsmooth domains. J. Analyse Math. 113, 53172 .

[GM76] M. L. Gorbachuk and V. A. Mikhailets. 1976. Semibounded selfadjoint extensions of symmetric operators. Dokl. Akad. Nauk SSSR 226. English translation in Soviet Math. Doklady 17, 185-186.

[GG91] V. I. Gorbachuk and M. L. Gorbachuk. Boundary value problems for operator differential equations. Kluwer, Dordrecht 1991.

[G68] G. Grubb. 1968. A characterization of the non-local boundary value problems associated with an elliptic operator Ann. Scuola Norm. Sup. Pisa 22, 425-513.

[G70] G. Grubb. 1970. Les problèmes aux limites généraux d'un opérateur elliptique, provenant de la théorie variationnelle. Bull. Sc. Math. 94, 113-157.

[G71] G. Grubb. 1971. On coerciveness and semiboundedness of general boundary value problems. Israel J. Math. 10, 32-95.

[G73] G. Grubb. 1973. Weakly semibounded boundary problems and sesquilinear forms. Ann. Inst. Fourier Grenoble 23, 145-194.

[G74] G. Grubb. 1974. Properties of normal boundary problems for elliptic even-order systems. Ann. Scuola Norm. Sup. Pisa 1 (ser.IV), 1-61.

[G83] G. Grubb. 1983. Spectral asymptotics for the "soft" selfadjoint extension of a symmetric elliptic differential operator. J. Operator Theory 10, 9-20.

[G84] G. Grubb. 1984. Singular Green operators and their spectral asymptotics. Duke Math. J. $\mathbf{5 1}, 477-528$.

[G84a] G. Grubb. 1984. Remarks on trace extensions for exterior boundary problems Comm. Partial Diff. Equ. 9, 231-270.

[G96] G. Grubb. Functional Calculus of Pseudodifferential Boundary Problems, Progress in Math. vol. 65, Second Edition. Birkhäuser, Boston 1996.

[G08] G. Grubb. 2008. Krein resolvent formulas for elliptic boundary problems in nonsmooth domains. Rend. Sem. Mat. Univ. Pol. Torino 66, 13-39.

[G09] G. Grubb. Distributions and operators. Graduate Texts in Mathematics 252. Springer, New York 2009.

[G11] G. Grubb. 2011. Perturbation of essential spectra of exterior elliptic problems. Applicable Analysis 90, 103-123.

[G11a] G. Grubb. Krein-like extensions and the lower boundedness problem for elliptic operators on exterior domains. arXiv:1002.4549, to appear.

[G11b] G. Grubb. 2011. Spectral asymptotics for Robin problems with a discontinuous coefficient. J. Spectral Theory 1, 155-177.

[G11c] G. Grubb. 2011. The mixed boundary value problem, Krein resolvent formulas and spectral asymptotic estimates. J. Math. Anal. Appl. 382, 339-263.

[H63] L. Hörmander. Linear Partial Differential Operators, Grundlehren Math. Wiss. vol. 116. Springer Verlag, Berlin 1963.

[H65] L. Hörmander. 1965. Pseudo-differential operators. Comm. Pure Appl. Math. 18, 501-517.

[H68] L. Hörmander. 1968. The spectral function of an elliptic operator. Acta Math 121, 193-218.

[H71] L. Hörmander. 1971. Fourier integral operators I. Acta Math. 127, 79-183.

[H85] L. Hörmander. The Analysis of Linear Partial Differential Operators III, Pseudo-differential Operators, Grundlehren Math. Wiss. vol. 274. Springer Verlag, Berlin 1985.

[I82] V. Ja. Ivrii. 1982. Accurate spectral asymptotics for elliptic operators that act in vedtor bundles. Functional Analysis Prilozhen 16 no. 2, 30-38, English translation in Functional Analysis Appl. 16 (1983), 101-108.

[I84] V. Ivrii. Precise spectral asymptotics for elliptic operators acting in fiberings over manifolds with boundary. Lecture Notes in Mathematics, 1100. Springer-Verlag, Berlin 1984.

[I91] V. Ivrii. Microlocal Analysis and Precise Spectral Asymptotics. Springer-Verlag, Berlin 1991.

[KN65] J. J. Kohn and L. Nirenberg. 1965. An algebra of pseudo-differential operators. Comm. Pure Appl. Math. 18, 269-305.

[K75] A. N. Kočuber. 1975. Extensions of symmetric operators and symmetric binary relations. Math. Notes (1) 17, 25-28.

[KK04] N.D. Kopachevskiı̌ and S.G. Krĕn. 2004. Abstract Green formula for a triple of Hilbert spaces, abstract boundary value and spectral problems. Ukr. Math. Bull. 1, 77-105.

[K47] M. G. Krel̆n. 1947. The theory of self-adjoint extensions of semi-bounded Hermitian transformations and its applications. I. Mat. Sbornik 20 431-495. (In Russian.)

[L85] O. Ladyshenskaya. The Boundary Value Problems of Mathematical Physics. SpringerVerlag, New York 1985

[LM68] J.-L. Lions and E. Magenes. Problèmes aux limites non homogènes et applications, 1. Éditions Dunod, Paris 1968. 
[L53] Ya. B. Lopatinskiü. 1953. On a method of reducing boundary problems for a system of differential equations of elliptic type to regular integral equations. Ukrain. Mat. Zb. 5, 123151.

[LS83] V. E. Lyantze and O. G. Storozh. Methods of the Theory of Unbounded Operators. Naukova Dumka, Kiev 1983. (In Russian.)

[M10] M. M. Malamud. Spectral theory of elliptic operators in exterior domains. Russian J. Math. Phys. 17, 96-125.

[MM02] M. M. Malamud and V. I. Mogilevskii. 2002. Krĕn type formula for canonical resolvents of dual pairs of linear relations. Methods Funct. Anal. Topology (4) 8, 72-100.

[M48] S. G. Mihlin. 1948. Singular integral equations. Uspehi Matem. Nauk (N.S.) 3, 29-112. English translation in Amer. Math. Soc. Translations 24 (1950), 116 pp.

[N29] J. von Neumann. 1929. Allgemeine Eigenwerttheorie Hermitescher Funktionaloperatoren. Math. Ann. 102, 49-131.

[N55] L. Nirenberg. 1955. Remarks on strongly elliptic partial differential equations. Comm. Pure Appl. Math. 8, 649-675.

[PR09] A. Posilicano and L. Raimondi. 2009. Krein's resolvent formula for self-adjoint extensions of symmetric second-order elliptic differential operators. J. Phys. A 42 015204, 11 pp.

[RS82] S. Rempel and B.-W. Schulze. Index Theory of Elliptic Boundary Problems. AkademieVerlag, Berlin 1982.

[R07] V. Ryzhov. 2007. A general boundary value problem and its Weyl function. Opuscula Math. 27 305-331.

[SV97] Yu. Safarov and D. Vassiliev. The Asymptotic Distribution of Eigenvalues of Partial Differential Operators. Translated from the Russian manuscript by the authors. Translations of Mathematical Monographs, 155. American Mathematical Society, Providence, R.I. 1997.

[Sch50] L. Schwartz. Théorie des distributions I-II. Hermann, Paris 1950-51.

[Se65] R. T. Seeley. 1965. Refinement of the functional calculus of Calderon and Zygmund. Proc. Konikl. Nederl. Akad. Wetensch. 68, 521-531.

[Se78] R. T. Seeley. 1978. A sharp asymptotic remainder estimate for the eigenvalues of the Laplacian in a domain of $R^{3}$. Adv. in Math. 29, 244-269.

[Sh53] Z. Ya. Shapiro. 1953. On general boundary value problems of elliptic type. Isz. Akad. Nauk, Math. Ser. 17, 539-562.

[So50] S. L. Sobolev. Some applications of functional analysis in mathematical physics. Izdat. Leningrad. Gos. Univ., Leningrad 1950. English translation by F. E. Browder. Translations of Mathematical Monographs, 7, American Mathematical Society, Providence, R.I. 1963.

[T80] F. Treves. Introduction to Pseudodifferential and Fourier Integral Operators, 1-2. Plenum Press, New York 1980.

[V80] L. I. Vainerman. 1980. On extensions of closed operators in Hilbert space. Math. Notes 28, $871-875$.

[V52] M. I. Vishik. 1952. On general boundary value problems for elliptic differential operators Trudy Mosc. Mat. Obsv. 1, 187-246. English translation in Amer. Math. Soc. Transl. (2) 24 (1963) 107-172.

[W12] H. Weyl. 1912. Das asymptotische Verteilungsgesetz der Eigenwerte linearer partieller Differentialgleichungen (mit einer Anwendung auf die Theorie der Hohlraumstrahlung). Math. Ann. 71, 441-479.

Department of Mathematical Sciences, Universitetsparken 5, DK-2100 Copenhagen, DENMARK, E-MAIL grubb@math.ku.dk 